ESAIM: M2AN 49 (2015) 641-662

DOI: $10.1051 / \mathrm{m} 2 \mathrm{an} / 2014053$
ESAIM: Mathematical Modelling and Numerical Analysis

www.esaim-m2an.org

\title{
THE REDUCED ORDER NS- $\alpha$ MODEL FOR INCOMPRESSIBLE FLOW: THEORY, NUMERICAL ANALYSIS AND BENCHMARK TESTING ${ }^{*, * *}, * * *$
}

\author{
Victoria M. Cuff ${ }^{1}$, Argus A. Dunca ${ }^{2}$, Carolina C. Manica ${ }^{3}$ \\ AND LEO G. REBHOLZ ${ }^{1}$
}

\begin{abstract}
This paper introduces a new, reduced-order NS- $\alpha$ ( $\mathrm{rNS}-\alpha)$ model for the purpose of efficient, stable, and accurate simulations of incompressible flow problems on coarse meshes. We motivate the new model by discussing the difficulties in efficient and stable algorithm construction for the usual NS- $\alpha$ model, and then derive rNS- $\alpha$ by using deconvolution as an approximation to the filter inverse, which reduces the fourth order NS- $\alpha$ formulation to a second order model. After proving the new model is well-posed, we propose a $C^{0}$ finite element spatial discretization together with an IMEX BDF2 timestepping to create a linearized algorithm that decouples the conservation of mass and momentum equations from the filtering. We rigorously prove the algorithm is well-posed, and provided a very mild timestep restriction, is also stable and converges optimally to the model solution. Finally, we give results of several benchmark computations that confirm the theory and show the proposed model/scheme is effective at efficiently finding accurate coarse mesh solutions to flow problems.
\end{abstract}

Mathematics Subject Classification. 65N30, 76D05, 76F65.

Received January 8, 2014. Revised June 10, 2014

Published online 3 April 2015.

\section{INTRODUCTION}

The NS- $\alpha$ model for fluid flow simulation has become very popular in recent years as a tool to accurately predict the larger scales of fluid flow on much coarser meshes than direct numerical simulation (DNS) requires. It has been used successively for coarse mesh simulations of turbulent and transitional flow simulation $[7,8,31,34]$, ocean simulation [20], and magnetohydrodynamic flow simulation [35], to cite just a few examples. We consider

Keywords and phrases. Large Eddy Simulation, approximate deconvolution, finite element method, turbulent channel flow.

* Partially supported by NSF grant DMS1112593.

** Partially supported by Conselho Nacional de Pesquisa e Desenvolvimento (CNPq), Brazil.

*** Partially supported by NSF grant DMS1112593.

1 Department of Mathematical Sciences, Clemson University, USA. vcuff@clemson.edu; rebholz@clemson.edu

2 Department of Mathematics and Computer Science, Politehnica University of Bucharest, Romania. argus_dunca@yahoo.com

3 Departamento de Matematica Pura e Aplicada, UFRGS, Porto Alegre, RS 91509-900, Brazil. carolina.manica@ufrgs.br 
the model for incompressible, Newtonian fluids in a domain $\Omega$, in which case the model takes the form

$$
\begin{aligned}
v_{t}+(\nabla \times v) \times \bar{v}+\nabla p-\nu \Delta v & =f, \\
\nabla \cdot v=\nabla \cdot \bar{v} & =0, \\
-\alpha^{2} \Delta \bar{v}+\bar{v}-v & =0,
\end{aligned}
$$

where $v$ is considered velocity, $\bar{v}$ an averaged velocity (with filtering radius $\alpha>0$ ), p pressure, $f$ an external forcing, and $\nu$ is the kinematic viscosity. The system must also be equipped with appropriate initial and boundary conditions for $v$ and $\bar{v}$, and we note that the correct boundary conditions for $\bar{v}$ in the case of no-slip velocity $v$ is considered an open problem; herein we take $\bar{v}=0$ on boundaries where $v=0$, which provides reasonable numerical results.

This model is very attractive from a mathematical viewpoint, since it has been proven that it is well-posed [14], is frame invariant [17], adheres to Kelvin's circulation theorem [13], conserves a model energy and helicity [13], requires significantly fewer degrees of freedom than DNS for full resolution [13], and can accurately predict scalings of the turbulent boundary layer [9]. As most other fluid flow models do not have all (or even most) of these properties, NS- $\alpha$ is generally considered to be one of the most 'physically accurate' models. However, for $C^{0}$ finite element computations, there appears to be a drawback to NS- $\alpha$ in that stable and efficient timestepping algorithms do not seem to exist without severe restrictions on the timestep size; this is an open question which we discuss in more detail below.

The purpose of this paper is to introduce the reduced $N S-\alpha$ model ( $r N S-\alpha$ ), which will admit numerical algorithms with these important properties. The model is created by using an approximate deconvolution approximation to the inverse of the $\alpha$-filter in the fourth order NS- $\alpha$ system, yielding a second order model that can be discretized temporally with a BDF2 IMEX approach to both linearize the momentum equation and decouple the filtering and mass/momentum equations at each time step, but still provide stability and and optimal convergence with a very mild timestep restriction. We will derive the model, study its fundamental mathematical properties, propose a numerical algorithm for it, rigorously prove its stability and convergence properties, and test the model/method on some benchmark problems.

To discuss further the apparent inability to develop efficient and stable timestepping algorithms for NS- $\alpha$ when $C^{0}$ finite elements are used, consider the case of backward Euler timestepping and wall bounded flows. Denote the filtering operation also by $F$ (so that $F \phi:=\bar{\phi}$ ), and consider the scheme for (1.1):

$$
\frac{1}{\Delta t}\left(v^{n+1}-v^{n}\right)+\left(\nabla \times v^{n+1}\right) \times \overline{v^{n+1}}+\nabla p^{n+1}-\nu \Delta v^{n+1}=f^{n+1} .
$$

Multiplying the model by $\overline{v^{n+1}}$ and integrating over the domain will vanish both the nonlinear and pressure terms, then applying standard analysis (e.g. such as those in [39] for NS- $\alpha$ type models) provides

$$
\frac{1}{\Delta t}\left(\left\|F^{1 / 2} v^{n+1}\right\|^{2}-\left\|F^{1 / 2} v^{n}\right\|^{2}\right)+\nu\left\|\nabla F^{1 / 2} v^{n+1}\right\|^{2} \leq \nu^{-1}\left\|F^{1 / 2} f^{n+1}\right\|_{-1}^{2},
$$

where here and throughout, $(\cdot, \cdot)$ and $\|\cdot\|$ denote the $L^{2}(\Omega)$ inner product and norm, and $\|\cdot\|_{-1}$ denotes the $H^{-1}(\Omega)$ norm. By summing over timesteps $n=0$ to $n=M-1$ and assuming $\|F \phi\| \leq\|\phi\|$, a stability bound is achieved:

$$
\left\|v^{M}\right\|_{F}^{2}+\nu \Delta t \sum_{n=1}^{M}\left\|\nabla v^{n}\right\|_{F}^{2} \leq C,
$$

where $\|\phi\|_{F}:=(\phi, F \phi)^{1 / 2}$, with $C$ independent of $\alpha$ and the timestep $\Delta t$. This proves the scheme is unconditionally stable with respect to the timestep.

The problem with the above scheme is that it is not efficient, as it requires the solution of a 4 unknown-4 equation nonlinear system at each timestep. If a Newton method is used, then a fully coupled block, nonsymmetric linear system arises at each Newton iteration, and solving these linear problems is generally not practical. 
The alternative is to decouple the linear system with an iteration, but this leads to first order convergence, and thus many nonlinear iterations may be required at each timestep. Ideally, for efficiency purposes, we wish to perform a single linear solve of the (decoupled) mass/momentum system and a single (decoupled) filter system solve at each timestep, which can be accomplished by linearizing the nonlinearity at each time step by lagging the filtered term in the timestepping via the IMEX scheme

$$
\frac{1}{\Delta t}\left(v^{n+1}-v^{n}\right)+\left(\nabla \times v^{n+1}\right) \times \overline{v^{n}}+\nabla p^{n+1}-\nu \Delta v^{n+1}=f^{n+1} .
$$

However, it does not seem possible to prove this algorithm is stable without a severe timestep restriction. More specifically, it does not seem that one can do better with the timestep restriction than for a scheme that lags the entire nonlinearity in time, i.e. $\Delta t<C h^{3}$ in 3D [33].

An alternative way to develop an efficient and stable NS- $\alpha$ timestepping algorithm is to consider the fourthorder version of the model, which is created by writing the model in terms on $\bar{v}$ only. Using (1.3) in (1.1), and writing $w=\bar{v}$ gives the system

$$
\begin{aligned}
-\alpha^{2} \Delta w_{t}+w_{t}+(\nabla \times w) \times w-\alpha^{2}(\nabla \times \Delta w) \times w+\nabla p-\nu \Delta w+\alpha^{2} \nu \Delta^{2} w & =f, \\
\nabla \cdot w & =0 .
\end{aligned}
$$

For this system, the filtering equations are eliminated, and by lagging the curl terms in the nonlinearity, it is straight-forward to construct unconditionally stable timestepping algorithms by lagging the first terms in the nonlinearities. However, for finite element computations, the system (1.6)-(1.7) is not attractive, since it is a fourth order model and therefore requires boundary conditions for higher order derivatives of $w$, and either the use of $C^{1}$ elements or additional implicitly coupled auxiliary variables. Neither of these options allow for efficient computations for large scale 3D problems.

To produce a model that is amenable to large scale $C^{0}$ finite element computations, we propose an alteration of the fourth order NS- $\alpha$ model (1.6)-(1.7) that uses approximate deconvolution to reduce the fourth order model to a second order model. Thus, consider $D$ to be a deconvolution operator that is an approximate inverse to $F$, i.e. $D \approx F^{-1}=\left(-\alpha^{2} \Delta+I\right)$. Using this approximation in (1.6)-(1.7) creates a new model, which we call the rNS- $\alpha$ model:

$$
\begin{aligned}
-\alpha^{2} \Delta w_{t}+w_{t}+(\nabla \times D w) \times w+\nabla p-\nu \Delta D w & =f, \\
\nabla \cdot w & =0, \\
w(0) & =w_{0} .
\end{aligned}
$$

The most common deconvolution operator, and the one we use herein, is the van Cittert type (other types of deconvolution, such as multiscale [10] or Tikhonov [32] are also possible). Van Cittert approximate deconvolution is constructed by a first order Richardson algorithm using the filter $F$, which defines the family of deconvolution operators

$$
D_{N}=\sum_{n=0}^{N}(I-F)^{n} .
$$

We note the idea of using approximate deconvolution in computational fluid dynamics was recently pioneered by Stolz, Adams and Kleiser in $[1,2,43,44]$, who used it in a closure approximation for the spatially filtered Navier-Stokes equations (NSE) to create a Large Eddy Simulation (LES) model. Since then, it has been used to increase accuracy in $\alpha$ type regularization models by changing the regularization operation from $F$ to $D_{N} F$ [28], which provides higher order formal accuracy due to the relation [11]

$$
D_{N} \bar{\phi}-\phi=(-1)^{N+1} \alpha^{2 N+2} \Delta^{N+1} F^{N+1} \phi .
$$


More recently, approximate deconvolution was used successfully as a model reduction tool to aid in computational efficiency of timestepping methods for finite element discretizations of Stolz-Adams deconvolution turbulence models [15], and has also been part of many recent LES studies (see [6], and reference therein).

Herein, we propose and study $C^{0}$ finite element in spatial discretizations of (1.8)-(1.10) together with IMEX BDF2 timestepping methods that linearize the system at each timestep and decouple the filtering from the mass/momentum system. We first propose a family of such algorithms, and then prove they are well-posed, and stable and optimally convergent provided a very mild timestep restriction. For completeness, since the continuous level rNS- $\alpha$ model is new, we prove its well-posedness. Finally, we test the model/scheme on several benchmark flow problems, and find that it does an excellent job of predicting accurate solutions using coarse meshes where DNS of NSE fails.

\section{WELL-POSEDNESS OF RNS- $\alpha$}

Before proposing numerical algorithms for $\mathrm{rNS}-\alpha$, since it is a new model it is important to check its mathematical validity. For fluid flow models, this generally means to show it is well-posed in the case of periodic boundary conditions. While such boundary conditions are not relevant in most application problems, if the model is not well-posed in this (simplest) setting, there is no hope for it to be well-posed when realistic boundary conditions are present.

The reduced model (1.8) shares structural similarities with the Navier-Stokes-Voight (NS-Voight) model, see $[4,23,25,37,38]$, in that it employs the Voight regularization and that its nonlinearity and viscous terms can be handled in the continuous analysis similarly to [25] or [4]. In this section, we use the notations and mathematical setting of [25], i.e. denote $\Omega$ as the periodic box and $H^{s}(\Omega)$ to be the usual Sobolev space of fractional order $s$. We denote the $L^{2}(\Omega)$ inner product by $(\cdot, \cdot)$, let

$$
P=\left\{\varphi: \Omega \rightarrow \mathbb{R}^{d} \mid \varphi \text { is a vector valued trigonometric polynomial with } \nabla \cdot \varphi=0, \int_{\Omega} \varphi=0\right\}
$$

$H$ be the closure of $P$ in $L^{2}(\Omega)$, and $V$ the closure of $P$ in $H^{1}(\Omega)$.

We assume the approximate deconvolution operator $D: H^{s}(\Omega) \rightarrow H^{s}(\Omega)$ satisfies the conditions in [42]: it is linear, positive operator that commutes with differentiation and

$$
c_{1}\|v\|_{s} \leq\|D v\|_{s} \leq c_{2}\|v\|_{s}
$$

for some positive constants $c_{1}, c_{2}$. We note this condition is satisfied by both van Cittert approximate deconvolution and multiscale deconvolution.

Following $[4,25]$ we formulate the definition of a regular weak solution of model (1.8), and prove it is wellposed.

Definition 2.1. For given $w_{0} \in V, f \in C\left((-T, T), H^{-1}(\Omega)\right), T>0$, a function $w \in C^{1}([-T, T], V)$ is a regular weak solution of the model (1.8) if $w(0)=w_{0}$ and

$$
\left(I-\alpha^{2} \Delta\right) w_{t}+(\nabla \times D w) \times w+\nabla p-\nu \Delta D w=f
$$

in the sense of the dual space $V^{\prime}$.

Theorem 2.2 (existence and uniqueness of a regular weak solution). For given $w_{0} \in V, f \in C((-T, T)$, $\left.H^{-1}(\Omega)\right), \nabla \cdot f=0, T>0$, a unique regular weak solution of the model (1.8) exists. Moreover, if $w_{0} \in H^{m}(\Omega)$, $f \in C\left((-T, T), H^{m-2}(\Omega)\right)$ then $w \in C^{1}\left((-T, T), H^{m}(\Omega)\right)$ and

$$
\sup _{t \in[-T, T]}\|w(t)\| \leq C\left(f, w_{0}, \alpha, \nu, c_{1}, c_{2}, T\right) .
$$


Proof. The proof follows the classical lines of the Galerkin method, which can be used similarly as in [4] since a priori energy estimates can be derived for the model (1.8)-(1.10) in a similar way as in the NS-Voight case.

Indeed, the nonlinear term in model (1.8) has the following property (see, for example, [14], property (4) on p. 5)

$$
((\nabla \times D w) \times w, v)=((w \cdot \nabla) D w, v)-((v \cdot \nabla) D w, w)
$$

whenever $v, w \in V$. This implies that if $w$ is a weak solution of (1.8), then

$$
((\nabla \times D w) \times w, w)=((w \cdot \nabla) D w, w)-((w \cdot \nabla) D w, w)=0 .
$$

Therefore, if we multiply (2.2) by $w$, integrate and cancel the nonlinearity, we obtain

$$
\frac{1}{2} \frac{\mathrm{d}}{\mathrm{d} t}\left(\|w\|^{2}+\alpha^{2}\|\nabla w\|^{2}\right)+\nu(\nabla D w, \nabla w)=(f, w),
$$

which further gives (due to the positivity of $D$ )

$$
\frac{\mathrm{d}}{\mathrm{d} t}\left(\|w\|^{2}+\alpha^{2}\|\nabla w\|^{2}\right)+C_{1} \nu\|\nabla w\|^{2} \leq \frac{C_{2}}{\nu}\|f\|_{-1}^{2}
$$

where $C_{1}, C_{2}$ are general constants only depending on $c_{1}$, see inequality (2.1).

This allows construction of approximate solutions that are uniformly bounded in the space $L^{\infty}\left([-T, T], H^{1}(\Omega)\right)$, and similar arguments such as the ones in the proof of Theorem 2.1 in [4] allow construction of a unique weak solution $w$ of (1.8).

A bootstraping argument similar to the Navier-Stokes-Voight case proves the higher order regularity properties of the model.

Theorem 2.3 (estimates of the modeling error). We let $v_{0} \in H, f \in C\left(0, T ; H_{-1}(\Omega)\right)$ and we assume that the NS- $\alpha$ model (1.1) has a strong solution $v$ in the sense of Layton ([26], Def. 29, p. 141), lying in the space $L^{4}\left(0, T ; H^{1}(\Omega)\right)$. We let $w$ be the solution of the $r N S-\alpha(1.8)$ corresponding to the initial condition $w_{0}=\bar{v}_{0}$. Then the following estimate of the modeling error $\bar{v}-w$ in terms of the deconvolution error $v-D \bar{v}$ holds:

$$
\begin{aligned}
& \|\bar{v}-w\|_{L^{\infty}\left(0, T ; L^{2}\right)}^{2}+\alpha^{2}\|\nabla(\bar{v}-w)\|_{L^{\infty}\left(0, T ; L^{2}\right)}^{2}+\nu\|\nabla(\bar{v}-w)\|_{L^{2}\left(0, T ; L^{2}\right)}^{2} \\
& \quad \leq C\left(\|v-D \bar{v}\|_{L^{4}\left(0, T ; L^{3}\right)}^{2}+\|\nabla(v-D \bar{v})\|_{L^{2}\left(0, T ; L^{2}\right)}^{2}\right)
\end{aligned}
$$

where $C=C\left(\Omega, T, f, v_{0}, \nu, c_{1}, c_{2},\|\nabla v\|_{L^{4}\left(0, T ; L^{2}\right)}\right)$.

Proof. The solution $v$ to the NS- $\alpha$ satisfies

$$
v_{t}+(\nabla \times v) \times \bar{v}+\nabla p-\nu \Delta v=f
$$

i.e.

$$
\left(I-\alpha^{2} \Delta\right) \bar{v}_{t}+(\nabla \times D \bar{v}) \times \bar{v}+\nabla p-\nu \Delta D \bar{v}=f+(\nabla \times D \bar{v}) \times \bar{v}-(\nabla \times v) \times \bar{v}-\nu \Delta D \bar{v}+\nu \Delta v .
$$

Subtracting it from (2.2), letting $e=w-\bar{v}$ and setting $e$ as a test function in the resulting equation gives

$$
\begin{aligned}
\frac{1}{2} \frac{\mathrm{d}}{\mathrm{d} t}\left(\|e\|^{2}+\alpha^{2}\|\nabla e\|^{2}\right)+(\nabla \times D e) & \times \bar{v}, e)+\nu(\nabla D e, \nabla e) \\
= & (\nabla \times(v-D \bar{v})) \times \bar{v}, e)+\nu(\nabla(v-D \bar{v}), \nabla e) .
\end{aligned}
$$

Using formula (2.1) we find a $C_{1} \geq 0$ such that $C_{1}\|\nabla e\| \leq(\nabla D e, \nabla e)$. We then estimate the last term on the left side in formula (2.4) to get

$$
\begin{aligned}
&((\nabla \times D e) \times \bar{v}, e) \leq C\|\nabla D e\|_{L^{2}}\|\bar{v}\|_{L^{6}}\|e\|_{L^{3}} \\
& \leq C|| \nabla e\left|\|\mid\| \bar{v}\left\|_{H^{1}}\right\| e\left\|^{1 / 2}\right\| \nabla e\left\|^{1 / 2} \leq \frac{C}{\nu^{3}}\right\| v\left\|_{H^{1}}^{4}\right\| e\left\|^{2}+\frac{C_{1} \nu}{6}\right\| \nabla e \|^{2} .\right.
\end{aligned}
$$


The first term on the right side in formula (2.4) is first integrated by parts, then the Sobolev embedding $H^{1} \hookrightarrow L^{6}$ and Poincaré inequality are used to get

$$
(\nabla \times(v-D \bar{v})) \times \bar{v}, e) \leq C\|v-D \bar{v}\|_{L^{3}}\|\bar{v}\|_{H^{1}}\|\nabla e\| \leq \frac{C}{\nu}\|\bar{v}\|_{H^{1}}^{2}\|v-D \bar{v}\|_{L^{3}}^{2}+\frac{C_{1} \nu}{6}\|\nabla e\|^{2} .
$$

The last term in the error equation (2.4) is estimated as

$$
\nu(\nabla(v-D \bar{v}), \nabla e) \leq C \nu\|\| \nabla(v-D \bar{v})\left\|^{2}+\frac{C_{1} \nu}{6}\right\| \nabla e \|^{2} .
$$

Replacing in the error equation and integrating from 0 to $0<t \leq T$ gives

$$
\begin{aligned}
\|e(t)\|^{2}+\alpha^{2} \| \nabla e(t) & \left\|^{2}+C_{1} \nu \int_{0}^{t}\right\| \nabla e(s)\left\|^{2} \mathrm{~d} s \leq \frac{C}{\nu^{3}} \int_{0}^{t}\right\| v(s)\left\|_{H^{1}}^{4}\right\| e(s) \|^{2} \mathrm{~d} s \\
+ & \frac{C}{\nu}\|\nabla v\|_{L^{4}\left(0, T ; L^{2}\right)}^{2}\|v-D \bar{v}\|_{L^{4}\left(0, T ; L^{3}\right)}^{2}+C \nu\|\nabla(v-D \bar{v})\|_{L^{2}\left(0, T ; L^{2}\right)}^{2} .
\end{aligned}
$$

The Grönwall's inequality completes the proof.

Remark 2.4. The above estimates hold in more general conditions, i.e. $f \in L^{2}\left(0, T ; H^{-1}(\Omega)\right.$.

In this case the first steps of Theorem 2.2 will construct a unique solution $w \in C(0, T ; H) \cap L^{\infty}(0, T ; V)$ of the rNS- $\alpha$ model with $\frac{d w}{d t} \in L^{2}(0, T ; V), w(0)=w_{0}$ and satisfying

$$
\left(I-\alpha^{2} \Delta\right) w_{t}+(\nabla \times D w) \times w+\nabla p-\nu \Delta D w=f
$$

in the sense of $V^{\prime}$ for a.e. $t \in[0, T]$. The arguments in the previous theorem will apply also in this setting.

\section{DiscretizATiOn AND NUMERICAL ANALYSis}

In this section, we propose and analyze an efficient numerical scheme for approximating solutions to rNS- $\alpha$ using a $C^{0}$ finite element spatial discretization, and an implicit-explicit BDF2 timestepping scheme that linearizes the system at each timestep and decouples the filtering/deconvolution equations from the momentum/mass equations. After providing some preliminaries, we present the scheme, and then prove its stability, well-posedness, and convergence.

\subsection{Problem setting and preliminaries}

For the numerical scheme, we consider $\Omega$ to be a convex polyhedron in $\mathbb{R}^{d}, d=2$ or 3 . We will consider homogeneous Dirichlet boundary conditions for velocity, making the natural function spaces for velocity and pressure

$$
\begin{aligned}
& X:=H_{0}^{1}(\Omega)^{d}=\left\{v \in H^{1}(\Omega)^{d}, v=0 \text { on } \partial \Omega\right\}, \\
& Q:=L_{0}^{2}(\Omega)=\left\{q \in L^{2}(\Omega), \int_{\Omega} v \mathrm{~d} x=0\right\} .
\end{aligned}
$$

Denote by $\tau_{h}$ a regular, conforming triangulation/tetrahedralization of $\Omega$. Let $\left(X_{h}, Q_{h}\right) \subset(X, Q)$ be an inf-sup stable pair of discrete velocity-pressure spaces defined on $\tau_{h}$, which are defined to be piecewise polynomials on each element. Define the discretely divergence free subspace by

$$
V_{h}:=\left\{v_{h} \in X_{h},\left(\nabla \cdot v_{h}, q_{h}\right)=0 \forall q_{h} \in Q_{h}\right\} .
$$


Discrete filtering will be defined by the standard finite element discretization of the $\alpha$ filter and, in contrast to most stable discretizations of regularization models, it does not enforce a solenoidal constraint, which makes it much more efficient. It is defined by: Given $\phi \in L^{2}(\Omega)$, find $F_{h} \phi=\bar{\phi}^{h} \in X_{h}$ satisfying

$$
\alpha^{2}\left(\nabla \bar{\phi}^{h}, \nabla \chi_{h}\right)+\left(\bar{\phi}^{h}, \chi_{h}\right)=\left(\phi, \chi_{h}\right) \quad \forall \chi_{h} \in X_{h}
$$

This filter is well-known to satisfy the following bounds [15]:

$$
\begin{aligned}
\phi \in L^{2}(\Omega): & \left\|\bar{\phi}^{h}\right\| \leq\|\phi\|, \\
\phi \in X_{h}: & \left\|\nabla \bar{\phi}^{h}\right\| \leq\|\nabla \phi\|, \\
\phi \in H^{1}(\Omega): & \left\|\nabla \bar{\phi}^{h}\right\| \leq C\|\nabla \phi\|,
\end{aligned}
$$

where $C$ depends only on $\Omega$ and arises from the use in the proof of the discrete Laplacian and the stability of $L^{2}$ projections of $H^{1}$ functions into $X_{h}$ from [5].

An important lemma for the approximation of the continuous filter by the discrete filter is given in the following lemma.

Lemma 3.1. The error in the approximation of the $\alpha$-filter satisfies

$$
\alpha^{2}\left\|\nabla\left(\bar{w}-\bar{w}^{h}\right)\right\|^{2}+\left\|\bar{w}-\bar{w}^{h}\right\|^{2} \leq 2 \inf _{\chi_{h} \in X_{h}}\left(\alpha^{2}\left\|\nabla\left(\bar{w}-\chi_{h}\right)\right\|^{2}+\left\|\bar{w}-\chi_{h}\right\|^{2}\right)
$$

If the velocity-pressure spaces are chosen to be the Taylor-Hood $\left(P_{k}, P_{k-1}\right)$ or Scott-Vogelius $\left(P_{k}, P_{k-1}^{\text {disc }}\right)$ pairs, then we have that

$$
\alpha^{2}\left\|\nabla\left(\bar{w}-\bar{w}^{h}\right)\right\|^{2}+\left\|\bar{w}-\bar{w}^{h}\right\|^{2} \leq C\left(\alpha^{2} h^{2 k}+h^{2 k+2}\right)|\bar{w}|_{k+1}^{2} .
$$

Remark 3.2. The dependence of the seminorm $|\bar{w}|_{k+1}$ on $\alpha$ is discussed in [28]. In the case of periodic boundary conditions or if the normal components of higher order derivatives of $\bar{w}$ are zero, then there is no dependence on $\alpha$. Otherwise, we have that

$$
\begin{aligned}
& |\bar{w}|_{j} \leq C|w|_{j} \quad \text { for } \quad j=1,2 \\
& |\bar{w}|_{j} \leq C \alpha^{-1}|w|_{j} \text { for } \quad j=3,4 \\
& |\bar{w}|_{j} \leq C \alpha^{-2}|w|_{j} \quad \text { for } \quad j=5,6
\end{aligned}
$$

and so on.

Proof. Denote $e=\bar{w}-\bar{w}^{h}$. Then by the definitions of the continuous and discrete filters, we get that

$$
\alpha^{2}\left(\nabla e, \nabla v_{h}\right)+\left(e, v_{h}\right)=0 \quad \forall v_{h} \in X_{h}
$$

Writing $e=\left(\bar{w}-\chi_{h}\right)+\left(\chi_{h}-\bar{w}^{h}\right)=: \eta+\phi_{h}$, where $\chi_{h} \in X_{h}$ is arbitrary, after choosing $v_{h}=\phi_{h}$ we have that

$$
\alpha^{2}\left\|\nabla \phi_{h}\right\|^{2}+\|\phi\|^{2}=-\alpha^{2}\left(\nabla \eta, \nabla \phi_{h}\right)-\left(\eta, \phi_{h}\right)
$$

and thus

$$
\alpha^{2}\left\|\nabla \phi_{h}\right\|^{2}+\|\phi\|^{2} \leq \alpha^{2}\|\nabla \eta\|^{2}+\|\eta\|^{2} .
$$

The triangle inequality finishes the proof. 
Discrete van Cittert deconvolution is defined analogous to the continuous case, but using the discrete filter, and takes the form

$$
D_{N}^{h}=\sum_{n=0}^{N}\left(I-F_{h}\right)^{n}
$$

We note that as operators on $X_{h}$, both $F_{h}$ and $D_{N}^{h}$ are symmetric and positive, and $\left\|F_{h}\right\| \leq 1$.

Since $\|F\|<1,\left\|F_{h}\right\|<1$, it follows that $\left\|D_{N}\right\|<N+1,\left\|D_{N}^{h}\right\|<N+1$. Moreover, as shown in the proof of Lemma 2.14 in [29] (which is in essence a corollary of Lem. 3.1), if $X_{h}=P_{k}$ then

$$
\alpha\left\|\nabla\left(D_{N} \phi-D_{N}^{h} \phi\right)\right\|+\left\|D_{N} \phi-D_{N}^{h} \phi\right\| \leq C(N)\left(\alpha h^{k}+h^{k+1}\right) \sum_{n=1}^{N}\left|F^{n} \phi\right|_{k+1} .
$$

We define next an explicit filter operator, that filters known quantities exactly, but for unknown quantities, it filters instead a second order extrapolated approximation to the quantity. It is defined by

$$
\widetilde{F_{h}} w_{h}^{j}=F_{h} w_{h}^{j} \text { for } j=1,2, \ldots, n \text {, and } \widetilde{F_{h}} w_{h}^{n+1}:=F_{h}\left(2 w_{h}^{n}-w_{h}^{n-1}\right) .
$$

This operator defines an explicit deconvolution operator

$$
\widetilde{D_{N}^{h}} w_{h}^{n+1}=\sum_{n=0}^{N}\left(I-\widetilde{F_{h}}\right)^{n} .
$$

We now present an important identity from [15] relating $D_{N}^{h}$ and $\widetilde{D_{N}^{h}}$. It results from a simple expansion of the deconvolution operator $D_{N}^{h}$ and algebraic properties of van Cittert approximation deconvolution operators:

$$
\begin{aligned}
\widetilde{D_{N}^{h}} \phi^{n+1} & \\
= & \phi^{n+1}+\left(\phi^{n+1}-F_{h}\left(2 \phi^{n}-\phi^{n-1}\right)\right)+\left(\phi^{n+1}-2 F_{h}\left(2 \phi^{n}-\phi^{n-1}\right)+F_{h}^{2}\left(2 \phi^{n}-\phi^{n-1}\right)\right) \\
& +\ldots+\left(\phi_{n+1}+\sum_{i=1}^{N}(-1)^{i} \kappa_{i}^{N} F_{h}^{i}\left(2 \phi^{n}-\phi^{n-1}\right)\right) \\
& =D_{N}^{h} \phi^{n+1}+F_{h}\left(I+\left(2 I-F_{h}\right)+\ldots+\sum_{i=1}^{N}(-1)^{i} \kappa_{i}^{N} F_{h}^{i-1}\right)\left(\phi^{n+1}-2 \phi^{n}+\phi^{n-1}\right) \\
= & D_{N}^{h} \phi^{n+1}+F_{h}\left(D_{0}^{h}+D_{1}^{h}+\ldots+D_{N-1}^{h}\right)\left(\phi^{n+1}-2 \phi^{n}+\phi^{n-1}\right) \\
= & D_{N}^{h} \phi^{n+1}+\sum_{j=0}^{N-1} F_{h} D_{j}^{h}\left(\phi^{n+1}-2 \phi^{n}+\phi^{n-1}\right) .
\end{aligned}
$$

Finally, we are able to present the IMEX BDF2, $C^{0}$ finite element scheme for the rNS- $\alpha$ model.

Algorithm 3.3 (BDF2). Given endtime $T>0$, timestep $\Delta t>0$, forcing $f \in L^{\infty}\left(0, T ; H^{-1}(\Omega)\right)$, and initial velocities $w_{h}^{-1}, w_{h}^{0} \in V_{h}$, set $M=\frac{T}{\Delta t}$ and for $n=1,2, \ldots, M-1$, find $\left(w_{h}^{n+1}, q_{h}^{n+1}\right) \in\left(X_{h}, Q_{h}\right)$ satisfying for all $\left(v_{h}, r_{h}\right) \in\left(X_{h}, Q_{h}\right)$,

$$
\begin{aligned}
\frac{\alpha^{2}}{2 \Delta t}\left(\nabla\left(3 w_{h}^{n+1}-4 w_{h}^{n}+w_{h}^{n-1}\right), \nabla v_{h}\right)+\frac{1}{2 \Delta t}\left(3 w_{h}^{n+1}-4 w_{h}^{n}+w_{h}^{n-1}, v_{h}\right) & \\
+\left(\left(\nabla \times D_{N}^{h}\left(2 w_{h}^{n}-w_{h}^{n-1}\right)\right) \times w_{h}^{n+1}, v_{h}\right)-\left(q_{h}^{n+1}, \nabla \cdot v_{h}\right) & \\
+\nu\left(\nabla \widetilde{D_{N}^{h}} w_{h}^{n+1}, \nabla v_{h}\right) & =\left(f\left(t^{n+1}\right), v_{h}\right), \\
\left(\nabla \cdot w_{h}^{n+1}, r_{h}\right) & =0 .
\end{aligned}
$$

Remark 3.4. As is common with BDF2 and other two step methods, a first step using backward Euler (which is second order if only 1 step is taken) can provide a second initial condition. 


\subsection{Analysis of the scheme}

We now analyze the stability and convergence of Algorithm 3.3.

Lemma 3.5 (stability). Suppose that $\Delta t<\frac{\alpha^{2}}{4 C(N) \nu}$. Then solutions of Algorithm 3.3 satisfy

$$
\alpha^{2}\left\|\nabla w_{h}^{M}\right\|^{2}+\left\|w_{h}^{M}\right\|^{2}+\sum_{n=0}^{M-1}\left\|w_{h}^{n+1}-2 w_{h}^{n}+w_{h}^{n-1}\right\|^{2}+\nu \Delta t \sum_{n=1}^{M}\left\|\nabla w_{h}^{n}\right\|^{2} \leq C \nu^{-1}=C(\text { data }) .
$$

Moreover, since the scheme is linear at each timestep, we conclude that solutions to Algorithm 3.3 exist uniquely, and thus the algorithm is well-posed.

Remark 3.6. In settings where LES models such as rNS- $\alpha$ are used, it is usually the case that $\nu<\alpha$ or even $\nu \ll \alpha$. Moreover, generally we choose $N \leq 3$, in which case $C(N) \leq 6$. Also, typically $\alpha=O(h)$ or larger, in order for filtering to have a non-negligible effect. Thus in practice, the timestep restriction becomes $\Delta t<O(h)$, but if $\nu \ll \alpha$, then even this bound is pessimistic.

Proof. Choose $v_{h}=w_{h}^{n+1}$ in (3.3), which vanishes both the pressure and nonlinear terms. Then using the algebraic identity

$$
a(3 a-4 b+c)=\frac{1}{2}\left(a^{2}+(2 a-b)^{2}\right)-\frac{1}{2}\left(b^{2}+(2 b-c)^{2}\right)+\frac{1}{2}(a-2 b+c)^{2}
$$

gives

$$
\begin{array}{r}
\frac{\alpha^{2}}{4 \Delta t}\left(\left(\left\|\nabla w_{h}^{n+1}\right\|^{2}-\left\|\nabla w_{h}^{n}\right\|^{2}\right)+\left(\left\|\nabla\left(2 w_{h}^{n+1}-w_{h}^{n}\right)\right\|^{2}-\left\|\nabla\left(2 w_{h}^{n}-w_{h}^{n-1}\right)\right\|^{2}\right)+\left\|\nabla\left(w_{h}^{n+1}-2 w_{h}^{n}+w_{h}^{n-1}\right)\right\|^{2}\right) \\
+\frac{1}{4 \Delta t}\left(\left(\left\|w_{h}^{n+1}\right\|^{2}-\left\|w_{h}^{n}\right\|^{2}\right)+\left(\left\|2 w_{h}^{n+1}-w_{h}^{n}\right\|^{2}-\left\|2 w_{h}^{n}-w_{h}^{n-1}\right\|^{2}\right)+\left\|w_{h}^{n+1}-2 w_{h}^{n}+w_{h}^{n-1}\right\|^{2}\right) \\
+\nu\left(\nabla \widetilde{D_{N}^{h}} w_{h}^{n+1}, \nabla w_{h}^{n+1}\right)=\left(f\left(t^{n+1}\right), w_{h}^{n+1}\right) .
\end{array}
$$

For the viscous term, we first decompose it using (3.2) and Cauchy-Schwarz to get

$$
\begin{aligned}
\nu\left(\nabla \widetilde{D_{N}^{h}} w_{h}^{n+1}, \nabla w_{h}^{n+1}\right) & =\nu\left(\nabla D_{N}^{h} w_{h}^{n+1}, \nabla w_{h}^{n+1}\right)+\nu \sum_{j=0}^{N-1}\left(\nabla F_{h} D_{j}^{h}\left(w_{h}^{n+1}-2 w_{h}^{n}+w_{h}^{n-1}\right), \nabla w_{h}^{n+1}\right) \\
& \geq \nu\left\|\nabla\left(D_{N}^{h}\right)^{1 / 2} w_{h}^{n+1}\right\|^{2}-\nu \sum_{j=0}^{N-1}\left\|\nabla F_{h} D_{j}^{h}\left(w_{h}^{n+1}-2 w_{h}^{n}+w_{h}^{n-1}\right)\right\|\left\|\nabla w_{h}^{n+1}\right\| .
\end{aligned}
$$

Since $\left\|\nabla F_{h} \psi_{h}\right\| \leq\left\|\nabla \psi_{h}\right\|$ for all $\psi_{h} \in X_{h}$, we have that $\left\|\nabla F_{h} D_{j}^{h} \psi_{h}\right\| \leq C(j)\left\|\nabla \psi_{h}\right\|$ and $\left\|\nabla D_{N}^{h} \psi_{h}\right\| \geq 1$ for $\psi_{h} \in X_{h}$, and thus using this and Young's inequality gives

$$
\begin{aligned}
\nu\left(\nabla \widetilde{D_{N}^{h}} w_{h}^{n+1}, \nabla w_{h}^{n+1}\right) & \geq \nu\left\|\nabla\left(D_{N}^{h}\right)^{1 / 2} w_{h}^{n+1}\right\|^{2}-\nu C(N)\left\|\nabla\left(w_{h}^{n+1}-2 w_{h}^{n}+w_{h}^{n-1}\right)\right\|\left\|\nabla w_{h}^{n+1}\right\| \\
& \geq \frac{\nu}{2}\left\|\nabla w_{h}^{n+1}\right\|^{2}-C(N) \nu\left\|\nabla\left(w_{h}^{n+1}-2 w_{h}^{n}+w_{h}^{n-1}\right)\right\|^{2} .
\end{aligned}
$$

Combining (3.7) and (3.8), and reducing provides

$$
\begin{aligned}
& \frac{\alpha^{2}}{4 \Delta t}\left(\left(\left\|\nabla w_{h}^{n+1}\right\|^{2}-\left\|\nabla w_{h}^{n}\right\|^{2}\right)+\left(\left\|\nabla\left(2 w_{h}^{n+1}-w_{h}^{n}\right)\right\|^{2}-\left\|\nabla\left(2 w_{h}^{n}-w_{h}^{n-1}\right)\right\|^{2}\right)\right) \\
& +\frac{1}{4 \Delta t}\left(\left(\left\|w_{h}^{n+1}\right\|^{2}-\left\|w_{h}^{n}\right\|^{2}\right)+\left(\left\|2 w_{h}^{n+1}-w_{h}^{n}\right\|^{2}-\left\|2 w_{h}^{n}-w_{h}^{n-1}\right\|^{2}\right)+\left\|w_{h}^{n+1}-2 w_{h}^{n}+w_{h}^{n-1}\right\|^{2}\right) \\
& \quad+\left(\frac{\alpha^{2}}{4 \Delta t}-C(N) \nu\right)\left\|\nabla\left(w_{h}^{n+1}-2 w_{h}^{n}+w_{h}^{n-1}\right)\right\|^{2}+\frac{\nu}{4}\left\|\nabla w_{h}^{n+1}\right\|^{2} \leq \nu^{-1}\left\|f\left(t^{n+1}\right)\right\|_{-1}^{2} .
\end{aligned}
$$


Using the assumption of the timestep size, and then multiplying both sides by $4 \Delta t$ and summing over timesteps yields

$$
\begin{aligned}
& \alpha^{2}\left\|\nabla w_{h}^{M}\right\|^{2}+\alpha^{2}\left\|\nabla\left(2 w_{h}^{M}-w_{h}^{M-1}\right)\right\|^{2}+\left(\left\|w_{h}^{M}\right\|^{2}+\left\|2 w_{h}^{M}-w_{h}^{M-1}\right\|^{2}+\sum_{n=0}^{M-1}\left\|w_{h}^{n+1}-2 w_{h}^{n}+w_{h}^{n-1}\right\|^{2}\right) \\
& \quad+\nu \Delta t \sum_{n=1}^{M}\left\|\nabla w_{h}^{n}\right\|^{2} \leq \alpha^{2}\left\|\nabla w_{h}^{0}\right\|^{2}+\alpha^{2}\left\|\nabla\left(2 w_{h}^{0}-w_{h}^{-1}\right)\right\|^{2}+\left\|w_{h}^{0}\right\|^{2}+\left\|2 w_{h}^{0}-w_{h}^{-1}\right\|^{2}+4 \nu^{-1} \Delta t \sum_{n=1}^{M}\left\|f\left(t^{n}\right)\right\|_{-1}^{2},
\end{aligned}
$$

from which the result follows.

We now prove convergence of the scheme.

Theorem 3.7. Suppose $\left(X_{h}, Q_{h}\right)$ are chosen to be either $\left(P_{k}, P_{k-1}\right)$ Taylor-Hood elements or $\left(P_{k}, P_{k-1}^{\text {disc }}\right)$ ScottVogelius elements and suppose further that $(w, p)$ is a solution of $(1.8)-(1.10)$ for a given $\alpha>0, \nu>0$, with forcing $f \in L^{\infty}\left(0, T ; H^{-1}(\Omega)\right)$ and initial velocity $w_{0} \in V_{h}$, satisfying regularity criteria $w \in L^{\infty}\left(0, T ; H^{k+1}(\Omega) \cap\right.$ $\left.H^{3}(\Omega)\right), p \in L^{\infty}\left(0, T ; H^{k}(\Omega)\right), w_{t}, w_{t t}, w_{t t t} \in L^{\infty}\left(0, T ; H^{k+1}(\Omega) \cap H^{3}(\Omega)\right)$. Suppose further that the stability criterion for the timestep size is satisfied. Then the error of Algorithm 3.3 in approximating solutions to the $r N S-\alpha$ model satisfies

$$
\begin{aligned}
\left\|w(T)-w_{h}^{M}\right\|+\sum_{n=0}^{M-1} \| & \left(w\left(t^{n+1}\right)-2 w\left(t^{n}\right)+w\left(t^{n-1}\right)\right)-\left(w_{h}^{n+1}-2 w_{h}^{n}+w_{h}^{n-1}\right) \| \\
& +\alpha^{2}\left\|\nabla\left(w(T)-w_{h}^{M}\right)\right\|+\nu\left(\Delta t \sum_{n=1}^{M}\left\|\nabla\left(w\left(t^{n}\right)-w_{h}^{n}\right)\right\|^{2}\right)^{1 / 2} \leq C\left(\Delta t^{2}+h^{k}\right) .
\end{aligned}
$$

Remark 3.8. We note that the convergence proof can be adapted for any choice of LBB stable velocity-pressure pair. For example, if the $\left(P_{1}^{b}, P_{1}\right)$ mini element is used, we would get the estimate

$$
\begin{aligned}
\left\|w(T)-w_{h}^{M}\right\|+\sum_{n=0}^{M-1}\left\|\left(w\left(t^{n+1}\right)-2 w\left(t^{n}\right)+w\left(t^{n-1}\right)\right)-\left(w_{h}^{n+1}-2 w_{h}^{n}+w_{h}^{n-1}\right)\right\| \\
+\alpha^{2}\left\|\nabla\left(w(T)-w_{h}^{M}\right)\right\|+\nu\left(\Delta t \sum_{n=1}^{M}\left\|\nabla\left(w\left(t^{n}\right)-w_{h}^{n}\right)\right\|^{2}\right)^{1 / 2} \leq C\left(\Delta t^{2}+h\right) .
\end{aligned}
$$

Remark 3.9. We note that there is no restriction on the timestep size other than that required for stability. This is because the linearized algorithm allows for an alternate version of the discrete Gronwall inequality to be applied (discussed in [19]), which has no timestep restriction. As is common in analyses of nearly all NSErelated schemes, the application of Gronwall's lemma produces a large constant $C$ in the error bound, which is dependent on $\mathrm{e}^{\nu^{-1}}$ and thus could potentially be large in practice for turbulent flow problems after long times.

In our view, this is a weakness of the technique used (i.e. Gronwall's lemma), and it is widely believed that such Gronwall constants are non-optimal and overly pessimistic. We observed no evidence of such a large constant in our computations, which include channel flow around a cylinder with $\nu=0.001$ and was ran until $T=8$, and $R e_{\tau}=180$ turbulent channel flow which was run until $T=40$; in both cases no deterioration of results occurred after longer times, and the simulation results agreed very well with DNS benchmark data. 
Proof. At time $t^{n+1},(1.8)$ can be written as:

$$
\begin{aligned}
\frac{\alpha^{2}}{2 \Delta t}\left(\nabla \left(3 w^{n+1}-\right.\right. & \left.\left.4 w^{n}+w^{n-1}\right), \nabla v_{h}\right)+\frac{1}{2 \Delta t}\left(3 w^{n+1}-4 w^{n}+w^{n-1}, v_{h}\right) \\
+((\nabla & \left.\left.\times D_{N}^{h}\left(2 w^{n}-w^{n-1}\right)\right) \times w^{n+1}, v_{h}\right)-\left(p\left(t^{n+1}\right), \nabla \cdot v_{h}\right)+\nu\left(\nabla \widetilde{D_{N}^{h}} w^{n+1}, \nabla v_{h}\right) \\
= & \left(f\left(t^{n+1}\right), v_{h}\right)-\alpha^{2}\left(\nabla\left(w_{t}\left(t^{n+1}\right)-\frac{3 w^{n+1}-4 w^{n}+w^{n-1}}{2 \Delta t}\right), \nabla v_{h}\right) \\
- & \left(w_{t}\left(t^{n+1}\right)-\frac{3 w^{n+1}-4 w^{n}+w^{n-1}}{2 \Delta t}, v_{h}\right)-\left(\left(\nabla \times D_{N} w^{n+1}\right) \times w^{n+1}, v_{h}\right) \\
& +\left(\left(\nabla \times D_{N}^{h}\left(2 w^{n}-w^{n-1}\right)\right) \times w^{n+1}, v_{h}\right)+\nu\left(\nabla\left(D_{N} w^{n+1}-\widetilde{D_{N}^{h}} w^{n+1}\right), \nabla v_{h}\right),
\end{aligned}
$$

for all $v_{h} \in V_{h}$. Subtracting (3.3) from (3.13) and letting $\mathrm{e}^{n}=w^{n}-w_{h}^{n}$, we have for arbitrary $q_{h}^{n+1} \in Q_{h}$,

$$
\begin{aligned}
\frac{\alpha^{2}}{2 \Delta t}\left(\nabla \left(3 \mathrm{e}^{n+1}-\right.\right. & \left.\left.\left.4 \mathrm{e}^{n}+\mathrm{e}^{n-1}\right), \nabla v_{h}\right)+\frac{1}{2 \Delta t}\left(3 \mathrm{e}^{n+1}-4 \mathrm{e}^{n}+\mathrm{e}^{n-1}\right), v_{h}\right)-\left(p\left(t^{n+1}\right)-q_{h}^{n+1}, \nabla \cdot v_{h}\right) \\
- & \left(\nabla \times D_{N}^{h}\left(2 w_{h}^{n}-w_{h}^{n-1}\right) \times w_{h}^{n+1}, v_{h}\right)+\left(\nabla \times D_{N}^{h}\left(2 w^{n}-w^{n-1}\right) \times w^{n+1}, v_{h}\right) \\
+ & \nu\left(\nabla \widetilde{D_{N}^{h}} \mathrm{e}^{n+1}, \nabla v_{h}\right)=-\alpha^{2}\left(\nabla\left(w_{t}\left(t^{n+1}\right)-\frac{3 w^{n+1}-4 w^{n}+w^{n-1}}{2 \Delta t}\right), \nabla v_{h}\right) \\
& -\left(w_{t}\left(t^{n+1}\right)-\frac{3 w^{n+1}-4 w^{n}+w^{n-1}}{2 \Delta t}, v_{h}\right)-\left(\left(\nabla \times D_{N} w^{n+1}\right) \times w^{n+1}, v_{h}\right) \\
& \quad+\left(\left(\nabla \times D_{N}^{h}\left(2 w^{n}-w^{n-1}\right)\right) \times w^{n+1}, v_{h}\right)+\nu\left(\nabla\left(D_{N} w^{n+1}-\widetilde{D_{N}^{h}} w^{n+1}\right), \nabla v_{h}\right) .
\end{aligned}
$$

Decompose $\mathrm{e}^{n}=\left(w^{n}-\widetilde{w}^{n}\right)-\left(w_{h}^{n}-\widetilde{w}^{n}\right)=\eta^{n}-\phi_{h}^{n}$, where $\widetilde{w}^{n}$ is arbitrary in $V_{h}$, and choose the test function $v_{h}=\phi_{h}^{n+1}$. After (3.6) is used and the viscous term is handled in the same way as in the stability proof, we get

$$
\begin{gathered}
\frac{1}{4 \Delta t}\left(\left\|\phi_{h}^{n+1}\right\|^{2}-\left\|\phi_{h}^{n}\right\|^{2}+\left\|2 \phi_{h}^{n+1}-\phi_{h}^{n}\right\|^{2}-\left\|2 \phi_{h}^{n}-\phi_{h}^{n-1}\right\|^{2}+\left\|\phi_{h}^{n+1}-2 \phi_{h}^{n}+\phi_{h}^{n-1}\right\|^{2}\right) \\
+\frac{\alpha^{2}}{4 \Delta t}\left(\left\|\nabla \phi_{h}^{n+1}\right\|^{2}-\left\|\nabla \phi_{h}^{n}\right\|^{2}+\left\|\nabla\left(2 \phi_{h}^{n+1}-\phi_{h}^{n}\right)\right\|^{2}-\left\|\nabla\left(2 \phi_{h}^{n}-\phi_{h}^{n-1}\right)\right\|^{2}\right) \\
+\left(\frac{\alpha^{2}}{4 \Delta t}-C(N) \nu\right)\left\|\nabla\left(\phi_{h}^{n+1}-2 \phi_{h}^{n}+\phi_{h}^{n-1}\right)\right\|^{2}+\frac{\nu}{2}\left\|\nabla \phi_{h}^{n+1}\right\|^{2} \\
\leq\left|\left(w_{t}\left(t^{n+1}\right)-\frac{3 w^{n+1}-4 w^{n}+w^{n-1}}{2 \Delta t}, \phi_{h}^{n+1}\right)\right|+\alpha^{2}\left|\left(\nabla w_{t}\left(t^{n+1}\right)-\nabla \frac{3 w^{n+1}-4 w^{n}+w^{n-1}}{2 \Delta t}, \nabla \phi_{h}^{n+1}\right)\right| \\
+\left(p\left(t^{n+1}\right)-q_{h}^{n+1}, \nabla \cdot \phi_{h}^{n+1}\right)+\frac{\alpha^{2}}{2 \Delta t}\left(\nabla\left(3 \eta^{n+1}-4 \eta^{n}+\eta^{n-1}\right), \nabla \phi_{h}^{n+1}\right) \\
+\frac{1}{2 \Delta t}\left(3 \eta^{n+1}-4 \eta^{n}+\eta^{n-1}, \phi_{h}^{n+1}\right)+\left|\left(\nabla \times\left(D_{N} w^{n+1}-D_{N}^{h}\left(2 w^{n}-w^{n-1}\right)\right) \times w^{n+1}, \phi_{h}^{n+1}\right)\right| \\
+\nu\left(\nabla\left(D_{N}-D_{N}^{h}\right) w^{n+1}, \nabla \phi_{h}^{n+1}\right)-\nu\left(\nabla \widetilde{D_{N}^{h}} \eta^{n+1}, \nabla \phi_{h}^{n+1}\right)-\left(\left(\nabla \times D_{N}^{h}\left(2 w_{h}^{n}-w_{h}^{n-1}\right)\right) \times \eta^{n+1}, \phi_{h}^{n+1}\right) \\
-\left(\left(\nabla \times D_{N}^{h}\left(2 \phi_{h}^{n}-\phi_{h}^{n-1}\right)\right) \times w^{n+1}, \phi_{h}^{n+1}\right)-\left(\left(\nabla \times D_{N}^{h}\left(2 \eta^{n}-\eta^{n-1}\right)\right) \times w^{n+1}, \phi_{h}^{n+1}\right) . \quad(3.15)
\end{gathered}
$$

The first two terms on the right hand side are analyzed in a standard way, using Taylor series approximation, and Cauchy-Schwarz and Young's inequalities, along with Poincare' for the first of them. The fourth and fifth terms on the right hand side are also handled in a standard way, following [12]. Treating the pressure term in the 
usual way, and reducing using the smoothness assumption on $w_{t t t}$ and the timestep restriction reduces (3.15) to

$$
\begin{aligned}
\frac{1}{4 \Delta t}\left(\left\|\phi_{h}^{n+1}\right\|^{2}-\left\|\phi_{h}^{n}\right\|^{2}+\left\|2 \phi_{h}^{n+1}-\phi_{h}^{n}\right\|^{2}-\left\|2 \phi_{h}^{n}-\phi_{h}^{n-1}\right\|^{2}+\left\|\phi_{h}^{n+1}-2 \phi_{h}^{n}+\phi_{h}^{n-1}\right\|^{2}\right) \\
\quad+\frac{\alpha^{2}}{4 \Delta t}\left(\left\|\nabla \phi_{h}^{n+1}\right\|^{2}-\left\|\nabla \phi_{h}^{n}\right\|^{2}+\left\|\nabla\left(2 \phi_{h}^{n+1}-\phi_{h}^{n}\right)\right\|^{2}-\left\|\nabla\left(2 \phi_{h}^{n}-\phi_{h}^{n-1}\right)\right\|^{2}\right)+\frac{\nu}{4}\left\|\nabla \phi_{h}^{n+1}\right\|^{2} \\
\leq C \nu^{-1}\left(1+\alpha^{2}\right) \Delta t^{4}+4 \nu^{-1}\left\|p\left(t^{n+1}\right)-q_{h}^{n+1}\right\|^{2}+C \nu^{-1}\left(1+\alpha^{2}\right)\left(\left\|\nabla \eta_{t}^{n+1}\right\|^{2}+\left\|\nabla \eta_{t}^{n}\right\|^{2}\right) \\
\quad+C \nu^{-1}\left(1+\alpha^{2}\right) \int_{t^{n-1}}^{t^{n+1}}\left\|\nabla \eta_{t t}\right\|^{2} \mathrm{~d} t+\left|\left(\nabla \times\left(D_{N} w^{n+1}-D_{N}^{h}\left(2 w^{n}-w^{n-1}\right)\right) \times w^{n+1}, \phi_{h}^{n+1}\right)\right| \\
+\nu\left(\nabla\left(D_{N}-D_{N}^{h}\right) w^{n+1}, \nabla \phi_{h}^{n+1}\right)-\nu\left(\nabla \widetilde{D_{N}^{h}} \eta^{n+1}, \nabla \phi_{h}^{n+1}\right)-\left(\left(\nabla \times D_{N}^{h}\left(2 w_{h}^{n}-w_{h}^{n-1}\right)\right) \times \eta^{n+1}, \phi_{h}^{n+1}\right) \\
\quad-\left(\left(\nabla \times D_{N}^{h}\left(2 \phi_{h}^{n}-\phi_{h}^{n-1}\right)\right) \times w^{n+1}, \phi_{h}^{n+1}\right)-\left(\left(\nabla \times D_{N}^{h}\left(2 \eta^{n}-\eta^{n-1}\right)\right) \times w^{n+1}, \phi_{h}^{n+1}\right) .
\end{aligned}
$$

We majorize the remaining terms on the right hand side of (3.16) individually. For the first one, we first integrate by parts, apply Holder's inequality and then Agmon's, then Cauchy-Schwarz and Young, that $\left\|D_{N}\right\| \leq N+1$ [28], and finally smoothness assumptions of $w$ and (3.1),

$$
\begin{aligned}
\mid(\nabla \times & \left.\left(D_{N} w^{n+1}-D_{N}^{h}\left(2 w^{n}-w^{n-1}\right)\right) \times w^{n+1}, \phi_{h}^{n+1}\right) \mid \\
& \leq C\left\|D_{N} w^{n+1}-D_{N}^{h}\left(2 w^{n}-w^{n-1}\right)\right\|\left\|w^{n+1}\right\|_{H^{3}}\left\|\nabla \phi_{h}^{n+1}\right\| \\
& \leq C \nu^{-1}\left\|D_{N}\left(w^{n+1}-\left(2 w^{n}-w^{n-1}\right)\right)\right\|^{2}+C \nu^{-1}\left\|\left(D_{N}-D_{N}^{h}\right)\left(2 w^{n}-w^{n-1}\right)\right\|^{2}+\frac{\nu}{32}\left\|\nabla \phi_{h}^{n+1}\right\|^{2} \\
& \leq C(N) \nu^{-1} \Delta t^{4}+C(N) \nu^{-1}\left(\alpha^{2} h^{2 k}+h^{2 k+2}\right)+\frac{\nu}{32}\left\|\nabla \phi_{h}^{n+1}\right\|^{2} .
\end{aligned}
$$

For the next term on the right hand side of (3.16), we use Cauchy-Schwarz and Young's inequalities, and (3.1) along with smoothness assumptions of the true solution to get

$$
\begin{aligned}
\nu\left(\nabla\left(D_{N}-D_{N}^{h}\right) w^{n+1}, \nabla \phi_{h}^{n+1}\right) & \leq 4 \nu\left\|\nabla\left(D_{N}-D_{N}^{h}\right) w^{n+1}\right\|^{2}+\frac{\nu}{32}\left\|\nabla \phi_{h}^{n+1}\right\|^{2} \\
& \leq C(N) \nu\left(h^{2 k}+\alpha^{-2} h^{2 k+2}\right)+\frac{\nu}{32}\left\|\nabla \phi_{h}^{n+1}\right\|^{2} .
\end{aligned}
$$

The next term is majoried using Cauchy-Schwarz and Young's inequalities, definition of the IMEX deconvolution operator, and finally that $\left\|F_{h}\right\| \leq 1$ and $\left\|D_{j}^{h}\right\| \leq(j+1)$, we find

$$
\begin{aligned}
\nu\left(\nabla \widetilde{D_{N}^{h}} \eta^{n+1}, \nabla \phi_{h}^{n+1}\right) & \leq \frac{\nu}{32}\left\|\nabla \phi_{h}^{n+1}\right\|^{2}+4 \nu\left\|\nabla \widetilde{D_{N}^{h}} \eta^{n+1}\right\|^{2} \\
& \leq \frac{\nu}{32}\left\|\nabla \phi_{h}^{n+1}\right\|^{2}+C(N) \nu\left(\left\|\nabla \eta^{n+1}\right\|^{2}+\left\|\nabla \eta^{n}\right\|^{2}+\left\|\nabla \eta^{n-1}\right\|^{2}\right) .
\end{aligned}
$$

The next term from the right hand side of (3.16) is handled with Holder's inequality, Sobolev embedding $H^{1} \hookrightarrow L^{4}$, (not optimal but fitting our purpose), Young's inequality, and that $\left\|\nabla D_{N}^{h} w_{h}^{n}\right\| \leq(N+1)\left\|\nabla w_{h}^{n}\right\|$, which yields

$$
\begin{aligned}
-((\nabla & \left.\left.\times D_{N}^{h}\left(2 w_{h}^{n}-w_{h}^{n-1}\right)\right) \times \eta^{n+1}, \phi_{h}^{n+1}\right) \\
& \leq C\left\|\nabla D_{N}^{h}\left(2 w_{h}^{n}-w_{h}^{n-1}\right)\right\|\left\|\eta^{n+1}\right\|_{L^{4}}\left\|\phi_{h}^{n+1}\right\|_{L^{4}} \\
& \leq C\left\|\nabla D_{N}^{h}\left(2 w_{h}^{n}-w_{h}^{n-1}\right)\right\|\left\|\nabla \eta^{n+1}\right\|\left\|\nabla \phi_{h}^{n+1}\right\| \\
& \leq C(N) \nu^{-1}\left\|\nabla\left(2 w_{h}^{n}-w_{h}^{n-1}\right)\right\|^{2}\left\|\nabla \eta^{n+1}\right\|^{2}+\frac{\nu}{32}\left\|\nabla \phi_{h}^{n+1}\right\|^{2} \\
& \leq C(N) \nu^{-1}\left(\left\|\nabla w_{h}^{n}\right\|^{2}+\left\|\nabla w_{h}^{n-1}\right\|^{2}\right)\left\|\nabla \eta^{n+1}\right\|^{2}+\frac{\nu}{32}\left\|\nabla \phi_{h}^{n+1}\right\|^{2} .
\end{aligned}
$$


For the second to last term in (3.16), we first integrate by parts, then apply Holder's inequality, Poincaré, and the Sobolev embedding $H^{2} \hookrightarrow L^{\infty}$. Then using smoothness assumptions of the true solution, Young's inequality, and that $\left\|D_{N}^{h}\right\| \leq N+1$, we have that

$$
\begin{aligned}
-(( & \left.\left.\times D_{N}^{h}\left(2 \phi_{h}^{n}-\phi_{h}^{n-1}\right)\right) \times w^{n+1}, \phi_{h}^{n+1}\right) \\
& \leq C\left\|D_{N}^{h}\left(2 \phi_{h}^{n}-\phi_{h}^{n-1}\right)\right\|\left\|\nabla w^{n+1}\right\|_{L^{\infty}}\left\|\phi_{h}^{n+1}\right\|+C\left\|D_{N}^{h}\left(2 \phi_{h}^{n}-\phi_{h}^{n-1}\right)\right\|\left\|w^{n+1}\right\|_{L^{\infty}}\left\|\nabla \phi_{h}^{n+1}\right\| \\
& \leq C\left\|D_{N}^{h}\left(2 \phi_{h}^{n}-\phi_{h}^{n-1}\right)\right\|\left\|w^{n+1}\right\|_{H^{3}}\left\|\nabla \phi_{h}^{n+1}\right\| \\
& \leq C(N)\left\|2 \phi_{h}^{n}-\phi_{h}^{n-1}\right\|\left\|\nabla \phi_{h}^{n+1}\right\| \\
& \leq C(N) \nu^{-1}\left(\left\|\phi_{h}^{n}\right\|^{2}+\left\|\phi_{h}^{n-1}\right\|^{2}\right)+\frac{\nu}{32}\left\|\nabla \phi_{h}^{n+1}\right\|^{2} .
\end{aligned}
$$

Finally, for the last term in (3.16), we use similar techniques as the above inequalites to obtain

$$
\begin{aligned}
-((\nabla & \left.\left.\times D_{N}^{h}\left(2 \eta^{n}-\eta^{n-1}\right)\right) \times w^{n+1}, \phi_{h}^{n+1}\right) \\
& \leq C\left\|\nabla D_{N}^{h}\left(2 \eta^{n}-\eta^{n-1}\right)\right\|\left\|\nabla w^{n+1}\right\|\left\|\nabla \phi_{h}^{n+1}\right\| \\
& \leq C(N) \nu^{-1}\left\|\nabla 2 \eta^{n}-\eta^{n-1}\right\|^{2}+\frac{\nu}{32}\left\|\nabla \phi_{h}^{n+1}\right\|^{2} \\
& \leq C(N) \nu^{-1}\left(\left\|\nabla \eta^{n}\right\|^{2}+\left\|\nabla \eta^{n-1}\right\|^{2}\right)+\frac{\nu}{32}\left\|\nabla \phi_{h}^{n+1}\right\|^{2} .
\end{aligned}
$$

Combining (3.16)-(3.22), we get the estimate

$$
\begin{gathered}
\frac{1}{4 \Delta t}\left(\left\|\phi_{h}^{n+1}\right\|^{2}-\left\|\phi_{h}^{n}\right\|^{2}+\left\|2 \phi_{h}^{n+1}-\phi_{h}^{n}\right\|^{2}-\left\|2 \phi_{h}^{n}-\phi_{h}^{n-1}\right\|^{2}+\left\|\phi_{h}^{n+1}-2 \phi_{h}^{n}+\phi_{h}^{n-1}\right\|^{2}\right) \\
+\frac{\alpha^{2}}{4 \Delta t}\left(\left\|\nabla \phi_{h}^{n+1}\right\|^{2}-\left\|\nabla \phi_{h}^{n}\right\|^{2}+\left\|\nabla\left(2 \phi_{h}^{n+1}-\phi_{h}^{n}\right)\right\|^{2}-\left\|\nabla\left(2 \phi_{h}^{n}-\phi_{h}^{n-1}\right)\right\|^{2}\right)+\frac{\nu}{16}\left\|\nabla \phi_{h}^{n+1}\right\|^{2} \\
\leq C(N) \nu^{-1}\left(1+\alpha^{2}\right) \Delta t^{4}+4 \nu^{-1}\left\|p\left(t^{n+1}\right)-q_{h}^{n+1}\right\|^{2}+C \nu^{-1}\left(1+\alpha^{2}\right)\left(\left\|\nabla \eta_{t}^{n+1}\right\|^{2}+\left\|\nabla \eta_{t}^{n}\right\|^{2}+\left\|\nabla \eta_{t}^{n-1}\right\|^{2}\right) \\
+C(N) \nu^{-1}\left(\alpha^{2} h^{2 k}+h^{2 k+2}\right)+C(N) \nu\left(h^{2 k}+\alpha^{-2} h^{2 k+2}\right) \\
+C(N) \nu\left(\left\|\nabla \eta^{n+1}\right\|^{2}+\left\|\nabla \eta^{n}\right\|^{2}\right)+C(N) \nu^{-1}\left(\left\|\nabla w_{h}^{n}\right\|^{2}+\left\|\nabla w_{h}^{n-1}\right\|^{2}\right)\left\|\nabla \eta^{n+1}\right\|^{2} \\
+C \nu^{-1}\left(1+\alpha^{2}\right) \int_{t^{n-1}}^{t^{n+1}}\left\|\nabla \eta_{t t}\right\|^{2} \mathrm{~d} t+C(N) \nu^{-1}\left(\left\|\phi_{h}^{n}\right\|^{2}+\left\|\phi_{h}^{n-1}\right\|^{2}\right)+C(N) \nu^{-1}\left(\left\|\nabla \eta^{n}\right\|^{2}+\left\|\nabla \eta^{n-1}\right\|^{2}\right) .
\end{gathered}
$$

Next, we multiply both sides by $4 \Delta t$, sum over timesteps and reduce to get

$$
\begin{gathered}
\left\|\phi_{h}^{M}\right\|^{2}+\sum_{n=0}^{M-1}\left\|\phi_{h}^{n+1}-2 \phi_{h}^{n}+\phi_{h}^{n-1}\right\|^{2}+\alpha^{2}\left\|\nabla \phi_{h}^{M}\right\|^{2}+\frac{\nu \Delta t}{4} \sum_{n=1}^{M}\left\|\nabla \phi_{h}^{n}\right\|^{2} \leq \\
C(N) T \nu^{-1}\left(1+\alpha^{2}\right) \Delta t^{4}+4 \nu^{-1} \Delta t \sum_{n=1}^{M}\left\|p\left(t^{n}\right)-q_{h}^{n+1}\right\|^{2}+C \nu^{-1}\left(1+\alpha^{2}\right) \Delta t \sum_{n=1}^{M}\left\|\nabla \eta_{t}^{n}\right\|^{2} \\
+C(N) \nu^{-1} \Delta t \sum_{n=-1}^{M-1}\left(\alpha^{2} h^{2 k}+h^{2 k+2}\right)+C(N) \nu \Delta t \sum_{n=1}^{M}\left(h^{2 k}+\alpha^{-2} h^{2 k+2}\right) \\
+C(N) \nu^{-1} \Delta t \sum_{n=0}^{M-1}\left(\left\|\nabla w_{h}^{n}\right\|^{2}+\left\|\nabla w_{h}^{n-1}\right\|^{2}\right)\left\|\nabla \eta^{n+1}\right\|^{2}+C(N) \nu^{-1} \Delta t \sum_{n=1}^{M-1}\left\|\phi_{h}^{n}\right\|^{2} \\
\quad+C \nu^{-1}\left(1+\alpha^{2}\right) \int_{0}^{T}\left\|\nabla \eta_{t t}\right\|^{2}+C(N)\left(\nu^{-1}+\nu\right) \Delta t \sum_{n=1}^{M-1}\left\|\nabla \eta^{n}\right\|^{2} .
\end{gathered}
$$


Next, we use interpolation estimates for the $\eta$ terms on the right hand side of (3.24), Lemma 3.1 and the stability estimate (3.5) on the fourth from last term, which reduces (3.24) to

$$
\begin{aligned}
& \left\|\phi_{h}^{M}\right\|^{2}+\sum_{n=0}^{M-1}\left\|\phi_{h}^{n+1}-2 \phi_{h}^{n}+\phi_{h}^{n-1}\right\|^{2}+\alpha^{2}\left\|\nabla \phi_{h}^{M}\right\|^{2}+\nu \Delta t \sum_{n=1}^{M}\left\|\nabla \phi_{h}^{n}\right\|^{2} \leq \\
& C(N)\left(\nu^{-1}\left(1+\alpha^{2}\right) \Delta t^{4}+\left(\nu+\nu^{-1}+\nu^{-3}+\nu^{-1} \alpha^{2}\right) h^{2 k}+\left(\nu h^{2 k}+\nu \alpha^{-2} h^{2 k+2}+\nu^{-1} \alpha^{2} h^{2 k}+\nu^{-1} h^{2 k+2}\right)\right) \\
& +C(N) \nu^{-1} \Delta t \sum_{n=1}^{M-1}\left\|\phi_{h}^{n}\right\|^{2} \text {. }
\end{aligned}
$$

Applying the discrete Gronwall inequality from [19] that applies when the right hand side sum is only up to $M-1$ instead of $M$, by considering the integration terms to be $\left\|\phi_{h}^{n}\right\|^{2}$ (i.e. the first term on the left hand side and the last term on the right hand side, and noting that the rest of the terms fit the discrete Gronwall framework from [19]), we get that

$$
\begin{aligned}
& \left\|\phi_{h}^{M}\right\|^{2}+\sum_{n=0}^{M-1}\left\|\phi_{h}^{n+1}-2 \phi_{h}^{n}+\phi_{h}^{n-1}\right\|^{2}+\alpha^{2}\left\|\nabla \phi_{h}^{M}\right\|^{2}+\nu \Delta t \sum_{n=1}^{M}\left\|\nabla \phi_{h}^{n}\right\|^{2} \\
& \leq C_{*} \nu^{-1}\left(\left(1+\alpha^{2}\right) \Delta t^{4}+\left(\nu^{2}+1+\nu^{-2}+\alpha^{2}\right) h^{2 k}+\left(\nu^{2} h^{2 k}+\nu^{2} \alpha^{-2} h^{2 k+2}+\alpha^{2} h^{2 k}+h^{2 k+2}\right)\right) \\
& \leq C\left(\Delta t^{4}+h^{2 k}\right)
\end{aligned}
$$

where $C_{*}$ is a constant arising from the Gronwall inequality, and depends on $\exp \left(\nu^{-1}\right)$, although it is widely believed that this constant is a gross overestimate. From here, the triangle inequality and taking square roots completes the proof.

\section{NUMERICAL EXPERIMENTS}

In this section, we test the model/scheme on some benchmark problems. First, we confirm the predicted convergence rates on an analytical test problem with known solution. Then we test on a channel flow around a cylinder, and finally 3D turbulent channel flow.

\subsection{Verification of convergence rates}

Before computing convergence rates, we derive an exact solution for the reduced NS- $\alpha$ model. We then verify the predicted spatial and temporal convergence rates by calculating approximations to this solution on successively refined meshes and timestep sizes.

Following $[27,40]$, we derive the following exact Chorin-type velocity solution of reduced NS- $\alpha$ (i.e. it solves (1.8)-(1.10) with $f=0)$ :

$$
w=\left(\begin{array}{c}
-\cos (n \pi x) \sin (n \pi y) \\
\sin (n \pi x) \cos (n \pi y)
\end{array}\right) \mathrm{e}^{\frac{-2 C_{D_{N} n^{2} \pi^{2} \nu t}}{1+2 n^{2} \pi^{2} \alpha^{2}}},
$$

where $C_{D}$ is defined by noting that

$$
\bar{w}=\left(\frac{1}{1+2 n^{2} \pi^{2} \alpha^{2}}\right) w,
$$

and therefore by the definition of $D_{N}$, there is a positive constant $C_{D_{N}}$ satisfying

$$
D_{N} w=C_{D_{N}} w
$$


TABLE 1. Velocity errors and rates for a specified solution, using $\left(P_{1}^{b}, P_{1}\right)$ elements. Since the mesh width is cut by a factor of 4 with each refinement, a convergence rate of 2 is optimal and appears to be reached.

\begin{tabular}{cccc}
\hline$h$ & $\Delta t$ & $\left\|w-w_{h}\right\|_{2,1}$ & Rate \\
\hline $1 / 2$ & $1 / 2$ & $6.061 \mathrm{e}-1$ & - \\
$1 / 8$ & $1 / 4$ & $1.723 \mathrm{e}-1$ & 1.815 \\
$1 / 32$ & $1 / 8$ & $4.635 \mathrm{e}-2$ & 1.894 \\
$1 / 128$ & $1 / 16$ & $1.190 \mathrm{e}-2$ & 1.962 \\
\hline
\end{tabular}

The key to verifying (4.1) is a solution of (1.8)-(1.10) is using that $D_{N} w=C_{D_{N}} w$, since this implies with some vector identities that

$$
\begin{aligned}
\nabla \times\left(\left(\nabla \times D_{N} w\right) \times w\right) & =C_{D_{N}} \nabla \times((\nabla \times w) \times w) \\
& =C_{D_{N}}\left(\nabla \times w \cdot \nabla w-\frac{1}{2} \nabla \times \nabla|w|^{2}\right) \\
& =C_{D_{N}} \nabla \times(w \cdot \nabla w) \\
& =0,
\end{aligned}
$$

with the last equality holds by noticing that (4.1) is a Taylor-eddy solution (or by performing the calculation). This guarantees the existence of a pressure solution $q$ satisfying

$$
\nabla q=-C_{D_{N}}(\nabla \times w) \times w .
$$

From here, a calculation verifies that

$$
-\alpha^{2} \Delta w_{t}+w_{t}-\nu \Delta w=0
$$

which shows that $(w, q)$ defined by (4.1)-(4.2) is an exact solution for (1.8)-(1.10).

We now calculate approximations to the exact solution (4.1)-(4.2) of (1.8)-(1.10) on $\Omega=(0,1)^{2}$ and $0 \leq t \leq$ $T=0.1$, using Algorithm 3.3. We chose $f=0, N=1, n=1, \nu=1$, and $\alpha=\frac{1}{16}$, which yields $C_{D_{1}}=2-\frac{1}{1+2 \pi^{2} \alpha^{2}}$. Errors and rates for the scheme are computed, using solutions from successively refined uniform meshes and timesteps, with both the $\left(P_{2}, P_{1}\right)$ Taylor-Hood element and the $\left(P_{1}^{b}, P_{1}\right)$ mini element. Based on Theorem 3.7, we expect convergence in the energy norm for $\left(P_{2}, P_{1}\right)$ Taylor-Hood on the order of

$$
\left\|w-w_{h}\right\|_{2,1}:=\left\|w-w_{h}\right\|_{L^{2}\left(0, T ; H^{1}(\Omega)\right)}=O\left(h^{2}+\Delta t^{2}\right),
$$

and for the mini element,

$$
\left\|w-w_{h}\right\|_{2,1}=O\left(h+\Delta t^{2}\right) .
$$

For the mini element, we make successive refinements by cutting the mesh width by a factor of 4 , and cutting the timestep by a factor of 2 . With this refinement, the theorem predicts a convergence rate of 2 in the energy norm, which is what we observe from the errors and rates shown in Table 1. For $\left(P_{2}, P_{1}\right)$ Taylor-Hood, a convergence rate of 2 is predicted when we cut both the mesh width and the timestep by a factor of 2 at each refinement, which we observe to be the case from Table 2 .

\subsection{Turbulent channel flow with $\operatorname{Re}_{\tau}=180$}

Our next experiment is for the benchmark test of $R e_{\tau}=180$ turbulent channel flow. This problem was first studied by Kim et al. [24], and since then has been a very widely used benchmark test. An excellent description of the test problem setup can be found in [22], and the main details are as follows. The domain is a box

$$
\Omega=(-2 \pi, 2 \pi) \times(0,2) \times\left(-\frac{2 \pi}{3}, \frac{2 \pi}{3}\right),
$$


TABLE 2. Velocity errors and rates for a specified solution, using $\left(P_{2}, P_{1}\right)$ elements. The calculated rates are consistent with the optimal rate of 2 .

\begin{tabular}{cccc}
\hline$h$ & $\Delta t$ & $\left\|w-w_{h}\right\|_{2,1}$ & Rate \\
\hline $1 / 4$ & $1 / 2$ & $1.005 \mathrm{e}-1$ & - \\
$1 / 8$ & $1 / 4$ & $6.553 \mathrm{e}-2$ & 0.617 \\
$1 / 16$ & $1 / 8$ & $1.678 \mathrm{e}-2$ & 1.965 \\
$1 / 32$ & $1 / 16$ & $4.089 \mathrm{e}-3$ & 2.037 \\
$1 / 64$ & $1 / 32$ & $1.141 \mathrm{e}-3$ & 1.841 \\
\hline
\end{tabular}

and the kinematic viscosity is taken to be $\nu=\frac{1}{180}$. No slip boundary conditions are enforced at $y=0$ and $y=2$ (on the solid walls), and periodic boundary conditions are enforced on the remaining sides. The initial velocity is obtained by randomly perturbing the discrete mean velocity data $U_{\text {mean }}^{D N S}(y)$ from [36]; that is,

$$
\begin{aligned}
& w_{1}(x, y, z, 0)=U_{\text {mean }}^{D N S \operatorname{lin}}(y)+0.1 U_{\text {bulk }} \psi \\
& w_{2}(x, y, z, 0)=0.1 U_{\text {bulk }} \psi \\
& w_{3}(x, y, z, 0)=0.1 U_{\text {bulk }} \psi
\end{aligned}
$$

where $U_{\text {mean }}^{D N S, \operatorname{lin}}(y)$ is the linear interpolation of the mean DNS data of [36], $\psi$ is a random number from a uniform distribution on $[-1,1]$ (a new number is generated for each degree of freedom), and $U_{\text {bulk }}$ is calculated by

$$
U_{\mathrm{bulk}}=\int_{0}^{1} U_{\text {mean }}^{D N S \text { spline }}(y) \mathrm{d} y=15.6803
$$

where $U_{\text {mean }}^{D N S \text { spline }}(y)$ represents the cubic spline of $U_{\text {mean }}^{\text {DNS }}(y)$. Initial conditions $w_{h}^{0}=w_{h}^{-1}$ are taken to be the $L^{2}$ projection of $w(0)$ into $V_{h}$. The forcing is given by $f=\langle 1,0,0\rangle^{T}$, and following [22] is dynamically adjusted to maintain a nearly constant bulk velocity.

For the discretization, we first divide the box into rectangular cubes, with uniform grid spacing in the $x$ and $z$ directions $(3$ cell $\times 3$ cell discretization for both). For the grid spacing in the $y$ direction, we use the points $y_{n}=1-\cos (\pi n / 8)$, with $n=0,1, \ldots, 8$. Additionally, we add in two more $y$ points: $\{0.01,0.99\}$, to create a finer mesh near the solid walls. These cubes are then each divided uniformly into 6 tetrahedra, and then each tetrahedra is divided into 4 tetrahedra using a barycenter refinement. We choose $\left(\left(P_{3}\right)^{3}, P_{2}^{\text {disc }}\right)$ Scott-Vogelius finite elements, which provided 33,600 velocity degrees of freedom, which is dramatically less than the 6 million velocity degrees of freedom used in the DNS from [36]. We recall from [45] that these elements are known to be LBB stable on this type of mesh.

The resulting linear systems are solved directly with an artificial compressibility method, with parameter 1E-8, which provides solutions with mass conservation on the order of $\left\|\nabla \cdot w_{h}^{n}\right\|_{L^{2}} \approx 10^{-6}$, which is far better than can obtained with directly solving the coupled systems when Taylor-Hood elements are used (here we see only $\left.\left\|\nabla \cdot w_{h}^{n}\right\|_{L^{2}} \approx 10^{-1}\right)$. We note that our solver provides approximately the same level of mass conservation in solutions as does modified augmented Lagrangian preconditioned GMRES with tolerance $10^{-8}$ (i.e. the preconditioner/solver studied in $[3,18]$ ), but on this coarse mesh the direct solver is much faster.

We computed with Algorithm 3.3 to $T=40$. Spatial and temporal streamwise velocity averages are calculated from the nodal values, from time $T=20$ to $T=40$. Spatial averaging in $x$ and $z$ directions is performed by taking the mean value at all the nodes with the same $y$-value, since the discretization is uniform in these directions. These spatial averages at each timestep are then simply averaged in time. We computed with various choices of $\alpha$ and $\Delta t$, and the best results we found on this mesh was using the choice $\alpha=0.07$ (which is approximately the average mesh width) and $\Delta t=0.002$. These results are shown in Figure 1, along with the averaged DNS solution from [36], and it can be observed that the model's coarse mesh averaged velocity solution is in excellent agreement with that of the DNS. It is particularly interesting that the model gives such a good 

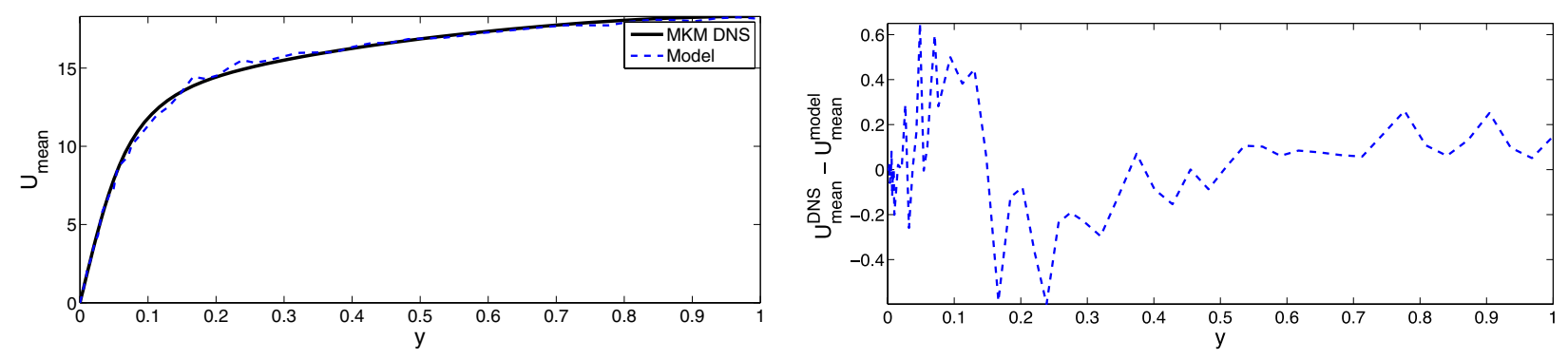

FIGURE 1. Shown above are the average velocity profile of the rNS- $\alpha$ solution with $\alpha=0.07$ and its difference from the DNS of Moser et al.
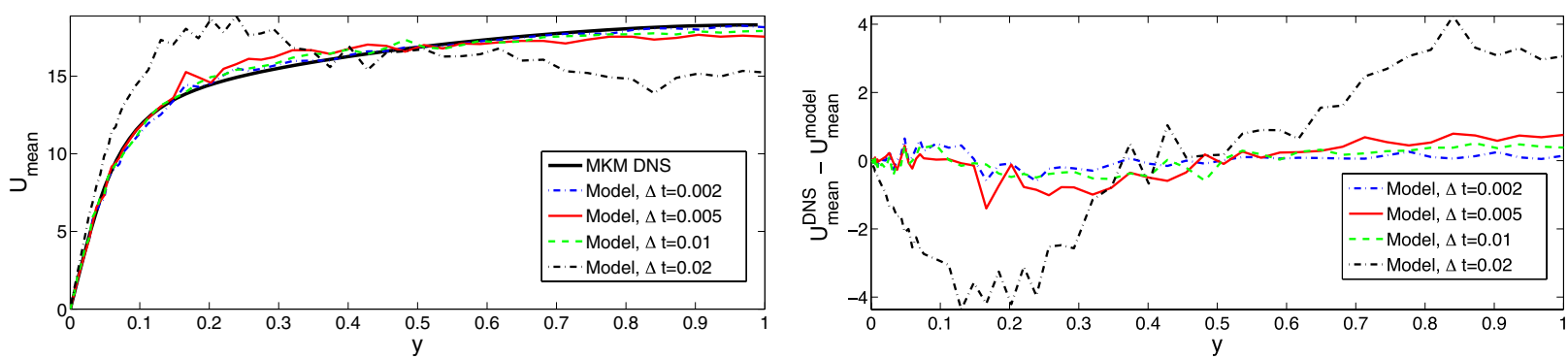

FiguRE 2. Shown above are the average velocity profiles, and their differences from the DNS, of the rNS- $\alpha$ solution with $\alpha=0.07$ and varying timestep sizes.

prediction near the wall, as it discussed in [22] and references therein how finite element and finite volume methods generally cannot correctly capture these statistics in coarse mesh simulations of turbulent channel flow compared to spectral methods, likely because their lower order of accuracy. To our knowledge, these are the best predictions found by any model on such a coarse mesh.

We also tested the model's sensitivity to the choice of $\alpha$ and $\Delta t$. Shown in Figure 2 are results using $\alpha=0.07$ with four choices of $\Delta t: 0.02,0.01,0.005$, and 0.002 . We observe that the $\Delta t=0.02$ solution is very inaccurate, and that the other three solutions have accuracy similar to each other, although the averaged velocity solution with $\Delta t=0.005$ seems to not be completely converged by $T=40$ (as evidenced by some minor oscillations in the figures). To test the sensitivity to $\alpha$, we computed using $\Delta t=0.002$ and with four choices of $\alpha: 0.15$, 0.10, 0.07 and 0.04. Results are shown in Figure 3, and show an interesting behavior of the model solution with varying $\alpha$ : as $\alpha$ decreases, so does the near-wall velocity. For $\alpha=0.15$, the near-wall velocity is under-predicted, and for $\alpha=0.04$, it is over-predicted. For the optimal $\alpha=0.07$ and for $\alpha=0.10$, the near-wall velocity is in good agreement with the DNS.

Remark 4.1. For comparison, we also tried to simulate turbulent channel flow using usual NS- $\alpha$, but with an equivalently efficient timestepping scheme, which we chose to be the IMEX scheme $\forall\left(v_{h}, q_{h}\right) \in\left(X_{h}, Q_{h}\right)$, find $\left(u_{h}^{n+1}, p_{h}^{n+1 / 2}\right) \in\left(X_{h}, Q_{h}\right)$ satisfying

$$
\begin{aligned}
\frac{1}{\Delta t}\left(u_{h}^{n+1}-u_{h}^{n}, v_{h}\right)+( & \left(\nabla \times u_{h}^{n+1 / 2}\right) \times{\left.\overline{\left(\frac{3}{2} u_{h}^{n}-\frac{1}{2} u_{h}^{n-1}\right)^{h}}, v_{h}\right)} \\
-\left(p_{h}^{n+1 / 2}, \nabla \cdot v_{h}\right)+\nu\left(\nabla u_{h}^{n+1 / 2}, \nabla v_{h}\right) & =\left(f\left(t^{n+1 / 2}\right), v_{h}\right) . \\
\left(\nabla \cdot u_{h}^{n+1}, q_{h}\right) & =0 .
\end{aligned}
$$



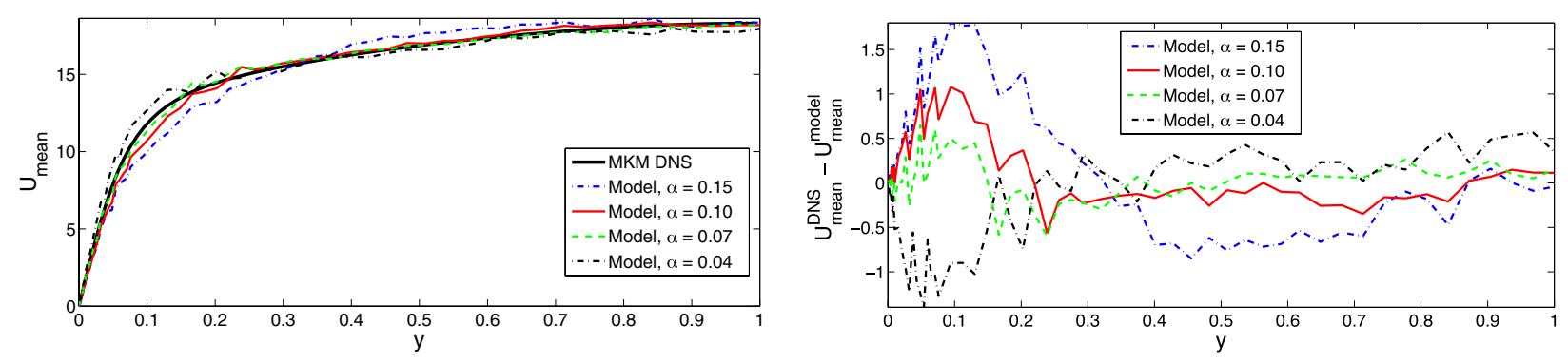

FiguRE 3. Shown above are the average velocity profiles, and their differences from the DNS, of the rNS- $\alpha$ solution with $\Delta t=0.002$ and varying $\alpha$.

This scheme is not known to have the same stability properties as the scheme studied herein (and we do not expect this to be true), but in our opinion this scheme is a reasonable choice for a comparison since it is second order, decouples the filtering, and has 1 mass/momentum solve at each timestep. The instability of this scheme is evidenced in the turbulent channel flow test problem, since the scheme (4.3)-(4.4) fails (blow up before $T=30$ ) for each $\alpha=0.03,0.04, \ldots, 0.25$, with $\Delta t=0.002$, on the same mesh as is used for the rNS- $\alpha$ computations above.

\subsection{D under resolved flow around a cylinder}

We now test the proposed model/scheme on a 2D benchmark problem of channel flow around a cylinder from $[21,41]$. The flow is driven by the interaction of the fluid with the cylinder, which is often an important scenario for industrial flows. This flow is not turbulent, but is very challenging for most numerical models/methods, especially on coarser meshes. The model performs quite well on this test problem, and with $\alpha$ chosen to be $O(h)$, relatively little sensitivity with respect to $\alpha$ is observed.

The domain is a $2.2 \times 0.41$ rectangle with a cylinder of radius 0.05 centered at $(0.2,0.2)$. We enforce no slip boundary conditions on all walls and the cylinder. The time dependent inflow and outflow profiles are set to be

$$
\begin{aligned}
& u_{1}(0, y, t)=u_{1}(2.2, y, t)=\frac{6}{0.41^{2}} \sin (\pi t / 8) y(0.41-y), \\
& u_{2}(0, y, t)=u_{2}(2.2, y, t)=0 .
\end{aligned}
$$

The flow is started from rest at $T=0$ and run until $T=8$, the external forcing is $f=0$, and the viscosity is set as $\nu=10^{-3}$, which produces an effective time dependent Reynolds number $0 \leq R e(t) \leq 100$. The correct physical behavior is for a vortex street to start to form behind the cylinder by $T=4$, and to persist through time $T=8$ [21]. The resolved Navier-Stokes solutions of [21,41] use upwards of 1 million total degrees of freedom and timesteps as small as $\Delta t=0.0001$.

Solutions were computed for the reduced NS- $\alpha$ model using Algorithm 3.3 with timestep $\Delta t=0.002$ and $\left(\left(P_{2}\right)^{2}, P_{1}^{\text {disc }}\right)$ Scott-Vogelius elements on a very coarse mesh that was created by making a barycenter refinement of a Delaunay generated mesh, which provided 5104 velocity degrees of freedom and 3708 pressure degrees of freedom and is shown in Figure 4. The choice of Scott-Vogelius elements is made because the reduced NS- $\alpha$ is implemented using the rotational form of the nonlinearity, and thus the 'pressure' represents an approximation to the Bernoulli pressure, which is significantly more complex than usual pressure. Since Scott-Vogelius elements provide divergence free velocities, they will completely remove the effect of large/complex pressures on the velocity solution [16]; we note that Taylor-Hood elements do allow for the pressure to adversely affect velocity accuracy, sometimes causing dramatic increase in error if a Bernoulli pressure is used [30]. Taking $N=2$ and varying $\alpha$ from 0.010 to 0.016 , (this range is approximately the mesh width near the cylinder), Algorithm 3.3 was used to compute solutions for rNS- $\alpha$ up to $T=8$. Vector plots of the velocity solutions are shown for 


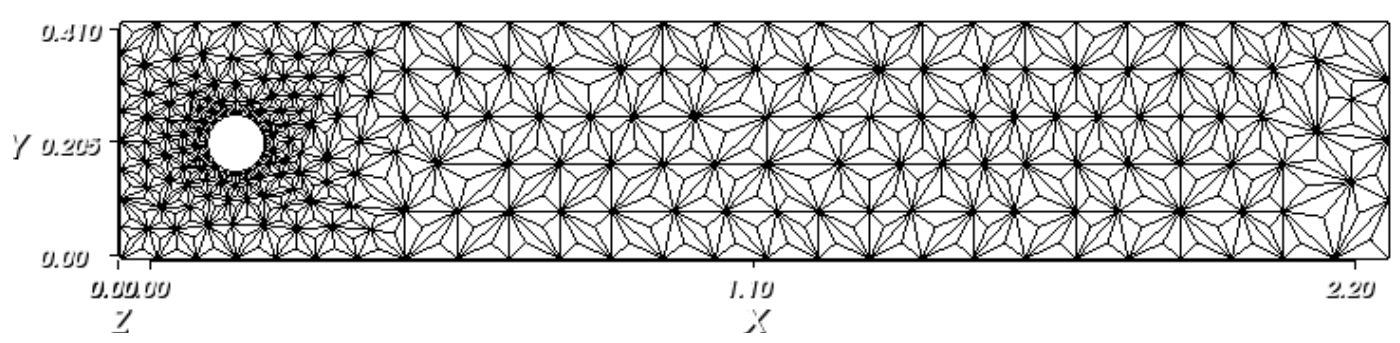

Figure 4. Shown above is the mesh used for the 2D flow around a cylinder computations.
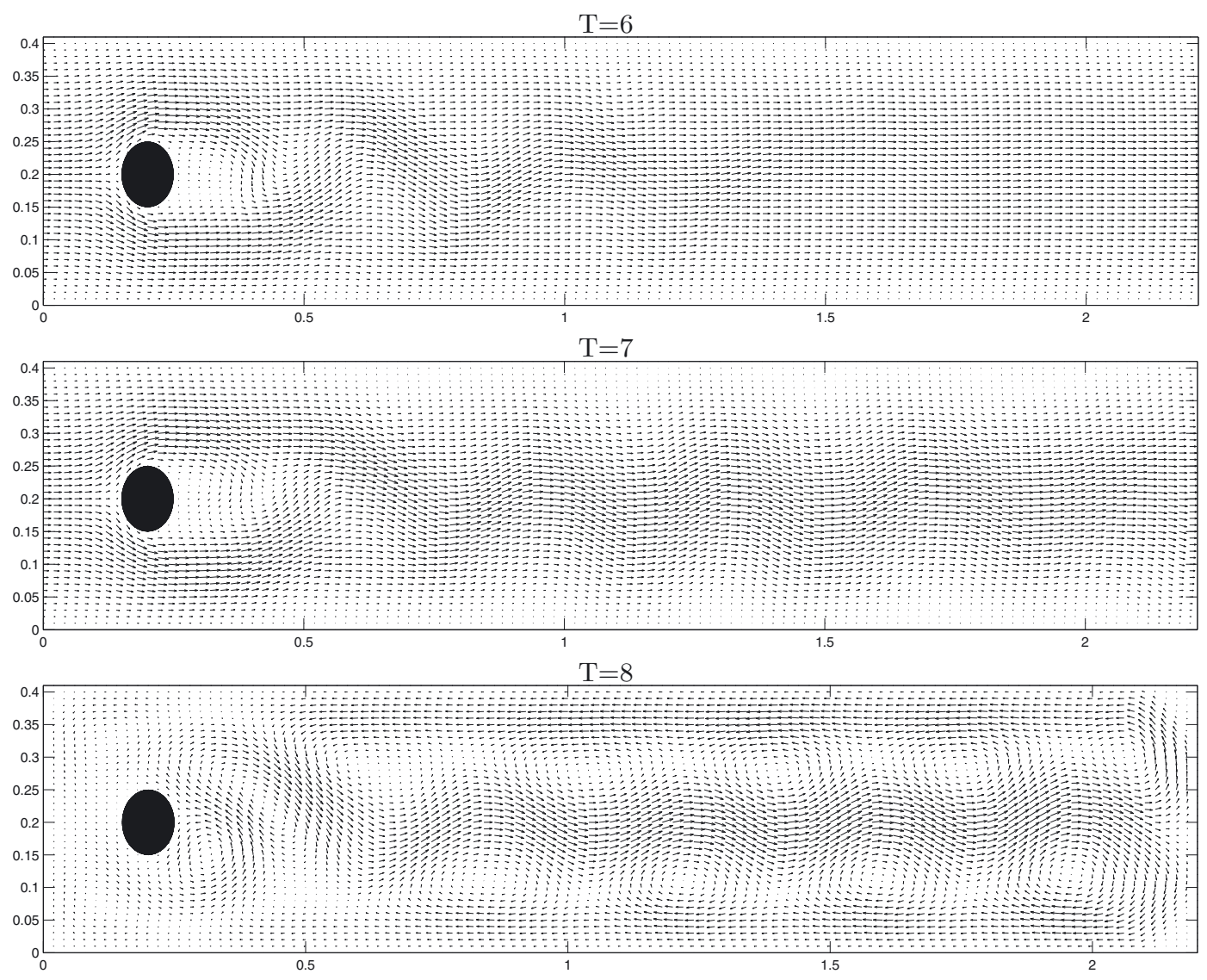

FiguRE 5. Shown above are the velocity vector plots for the 2D flow around a cylinder benchmark problem with Algorithm 3.3, at $T=6,7$, and 8 .

$T=6,7$, and 8 in Figure 5 for $\alpha=0.013$, but are indistinguishable from the plots from the other choices of $\alpha$. We note they agree with the fine mesh DNS solutions displayed in [21].

To further evaluate the coarse mesh model solutions, we also calculated values for the maximum $\operatorname{drag} c_{d, \max }$ and lift $c_{l, \max }$ coefficients at the cylinder, and compare them to those found in the resolved benchmark tests of $[21,41]$. The benchmark lift and drag coefficients for fully resolved NSE flow lie in the reference intervals

$$
c_{d, \max }^{\mathrm{ref}} \in[2.93,2.97], \quad c_{l, \max }^{\mathrm{ref}} \in[0.47,0.49] .
$$


TABLE 3. Max lift and drag coefficients for $2 \mathrm{~d}$ flow around a cylinder simulations on a coarse mesh with 5104 velocity degrees of freedom. Reference intervals from resolved simulations are $[2.93,2.97]$ for $c_{d, \max }$ and $[0.47,0.49]$ for $c_{l, \max }$.

\begin{tabular}{cccc}
\hline Model & $\alpha$ & $c_{d, \max }$ & $c_{l, \max }$ \\
\hline Navier-Stokes & - & 3.393 & 0.7499 \\
\hline $\mathrm{rNS}-\alpha$ & 0.010 & 3.044 & 0.524 \\
$\mathrm{rNS}-\alpha$ & 0.011 & 3.074 & 0.511 \\
$\mathrm{rNS}-\alpha$ & 0.012 & 3.107 & 0.506 \\
$\mathrm{rNS}-\alpha$ & 0.013 & 3.142 & 0.501 \\
$\mathrm{rNS}-\alpha$ & 0.014 & 3.180 & 0.497 \\
$\mathrm{rNS}-\alpha$ & 0.015 & 3.220 & 0.492 \\
$\mathrm{rNS}-\alpha$ & 0.016 & 3.264 & 0.487 \\
\hline
\end{tabular}

For comparison, we also give the max lift and drag coefficient values found on the same mesh, elements, and time step by the NSE discretized by the extrapolated Crank-Nicolson finite element method: $\forall\left(v_{h}, q_{h}\right) \in\left(X_{h}, Q_{h}\right)$,

$$
\begin{aligned}
\frac{1}{\Delta t}\left(u_{h}^{n+1}-u_{h}^{n}, v_{h}\right)+ & \left(\left(\nabla \times\left(\frac{3}{2} u_{h}^{n}-\frac{1}{2} u_{h}^{n-1}\right)\right) \times u_{h}^{n+1 / 2}, v_{h}\right) \\
-\left(p_{h}^{n+1 / 2}, \nabla \cdot v_{h}\right)+\nu\left(\nabla u_{h}^{n+1 / 2}, \nabla v_{h}\right) & =\left(f\left(t^{n+1 / 2}\right), v_{h}\right) . \\
\left(\nabla \cdot u_{h}^{n+1}, q_{h}\right) & =0 .
\end{aligned}
$$

To calculate lift and drag, we use the standard definitions (see e.g. [41]) but with the model solution velocities, and compute them using global integrals, following the derivations similar to those in [21] for NSE. The calculated coefficients are shown in Table 3. We observe that rNS- $\alpha$ provided much better prediction of the lift and drag coefficients than the NSE, for each of the chosen $\alpha$ 's, on this very coarse mesh. The predictions of the model are not in the reference intervals, but they are quite close, especially considering the coarseness of the mesh and the fact that lift and drag are defined using model velocity solutions, which are in some sense 'averaged velocities'. We observe further that there is mild sensitivity to the choice of $\alpha$; and as $\alpha$ increases, the drag increases and the lift decreases. To our knowledge, there are no results in the literature for this benchmark problem that are better than these for rNS- $\alpha$ on this coarse of a mesh.

\section{Conclusions}

We have derived a reduced order version of the NS- $\alpha$ model, and studied it from a continuous and numerical point of view. At the continuous level, we proved it is well-posed, and then we proposed a numerical scheme that is very efficient, and proved it is stable and optimally convergent provided a mild timestep restriction. Numerical experiments were given that confirmed the theory, and showed the model can give very good coarse mesh approximations to DNS results.

Regarding the choice of the parameter $\alpha$, this study has found the model to have mild sensitivity with respect to the parameter. In general, $\alpha=O(h)$ is a good choice, but finding the optimal constant is an open problem. In the flow around a cylinder test, solution plots with varying $\alpha$ were indistinguishable, and lift and drag varied slightly as $\alpha$ varied. For turbulent channel flow, $\alpha=0.07$ clearly gave the best results, but the $\alpha=0.04$ and $\alpha=0.10$ results were still good coarse mesh approximations. Still, a future sensitivity study of this model with respect to the parameter $\alpha$ is warranted.

Given the promising results of this first study of the $\mathrm{rNS}-\alpha$ model, there are many directions which could be followed both analytically and numerically, as well as applying this idea to coupled-physics problems. The authors plan to continue studying this model in each of these directions in future work. 


\section{REFERENCES}

[1] N.A. Adams and S. Stolz, On the Approximate Deconvolution procedure for LES. Phys. Fluids 2 (1999) $1699-1701$.

[2] N. Adams and S. Stolz, A subgrid-scale deconvolution approach for shock capturing. J. Comput. Phys. 178 (2002) $391-426$.

[3] M. Benzi and M. Olshanskii, An augmented Lagrangian-based approach to the Oseen problem. SIAM J. Sci. Comput. 28 (2006) 2095-2113.

[4] L.C. Berselli and L. Bisconti, On the structural stability of the Euler-Voight and Navier-Stokes-Voight models. Nonlinear Anal. 75 (2012) 117-130.

[5] J. Bramble, J. Pasciak and O. Steinbach, On the stability of the $L^{2}$ projection in $H^{1}(\Omega)$. Math. Comput. 71 (2002) $147-156$.

[6] T. Chacon and R. Lewandowski, Mathematical and numerical foundations of turbulence models and applications. Springer, New York (2014).

[7] S. Chen, C. Foias, D.D. Holm, E. Olson, E.S. Titi and S. Wynne, The Camassa-Holm equations as a closure model for turbulent channel and pipe flow. Phys. Rev. Lett. 81 (1998) 5338-5341.

[8] S. Chen, C. Foias, E. Olson, E.S. Titi and W. Wynne, A connection between the Camassa-Holm equations and turbulent flows in channels and pipes. Phys. Fluids 11 (1999) 2343-2353.

[9] A. Cheskidov, Boundary layer for the Navier-Stokes- $\alpha$ model of fluid turbulence. Arch. Ration. Mech. Anal. 172 (2004) $333-362$.

[10] A.A. Dunca, A two-level multiscale deconvolution method for the large eddy simulation of turbulent flows. Math. Models Methods Appl. Sci. 22 (2012) 1250001.

[11] A. Dunca and Y. Epshteyn, On the Stolz-Adams deconvolution model for the Large-Eddy simulation of turbulent flows. SIAM J. Math. Anal. 37 (2005) 1890-1902.

[12] V.J. Ervin and N. Heuer, Approximation of time-dependent, viscoelastic fluid flow: Crank-Nicolson, finite element approximation. Numer. Methods Partial Differ. Eq. 20 (2003) 248-283.

[13] C. Foias, D.D. Holm and E.S. Titi, The Navier-Stokes-alpha model of fluid turbulence. Physica D 152 (2001) 505-519.

[14] C. Foias, D.D. Holm and E.S. Titi, The three dimensional viscous Camassa-Holm equations, and their relation to the NavierStokes equations and turbulence theory. J. Dyn. Differ. Eq. 14 (2002) 1-35.

[15] K. Galvin, L. Rebholz and C. Trenchea, Efficient, unconditionally stable, and optimally accurate fe algorithms for approximate deconvolution models. SIAM J. Numer. Anal. 52 (2014) 678-707.

[16] V. Girault and P.-A. Raviart. Finite element methods for Navier-Stokes equations: theory and algorithms. Springer-Verlag (1986).

[17] J.L. Guermond, J.T. Oden and S. Prudhomme, An interpretation of the Navier-Stokes-alpha model as a frame-indifferent Leray regularization. Physica D 177 (2003) 23-30.

[18] T. Heister and G. Rapin, Efficient augmented Lagrangian-type preconditioning for the Oseen problem using grad-div stabilization. Int. J. Numer. Meth. Fluids 71 (2013) 118-134.

[19] J. Heywood and R. Rannacher, Finite element approximation of the nonstationary Navier-Stokes problem. Part IV: Error analysis for the second order time discretization. SIAM J. Numer. Anal. 2 (1990) 353-384.

[20] D. Holm and B.T. Nadiga, Modeling mesoscale turbulence in the barotropic double-gyre circulation. J. Phys. Oceanogr. 33 (2003) 2355-2365.

[21] V. John, Reference values for drag and lift of a two dimensional time-dependent flow around a cylinder. Int. J. Numer. Meth. Fluids 44 (2004) 777-788.

[22] V. John and M. Roland, Simulations of the turbulent channel flow at $R e_{\tau}=180$ with projection-based finite element variational multiscale methods. Int. J. Numer. Meth. Fluids 55 (2007) 407-429.

[23] V.K. Kalantarov and E.S. Titi, Global attractors and determining modes for the 3D Navier- Stokes-Voight equations. Chin. Ann. Math. Ser. B 30 (2009) 697-714.

[24] J. Kim, P. Moin and R. Moser, Turbulence statistics in fully developed channel flow at low Reynolds number. J. Fluid Mech. 177 (1987) 133-166.

[25] A. Larios and E.S. Titi, On the higher-order global regularity of the inviscid Voight regularization of the three-dimensional hydrodynamic models. Discrete Contin. Dyn. Syst. Ser. B 14 (2010) 603-627.

[26] W. Layton, Introduction to the Numerical Analysis of Incompressible Viscous Flows. SIAM (2008).

[27] W. Layton, On Taylor/eddy solutions of approximate deconvolution models of turbulence. Appl. Math. Lett. 24 (2011) 23-26.

[28] W. Layton and L. Rebholz, Approximate Deconvolution Models of Turbulence: Analysis, Phenomenology and Numerical Analysis. Springer-Verlag (2012).

[29] W. Layton, C. Manica, M. Neda and L. Rebholz, Numerical analysis and computational testing of a high accuracy Leraydeconvolution model of turbulence. Numer. Methods Partial Differ. Eq. 24 (2008) 555-582.

[30] W. Layton, C. Manica, M. Neda, M.A. Olshanskii and L. Rebholz, On the accuracy of the rotation form in simulations of the Navier-Stokes equations. J. Comput. Phys. 228 (2009) 3433-3447.

[31] E. Lunasin, S. Kurien, M. Taylor and E.S. Titi. A study of the Navier-Stokes-alpha model for two-dimensional turbulence. J. Turbulence 8 (2007) 751-778.

[32] C. Manica and I. Stanculescu, Numerical analysis of Leray-Tikhonov deconvolution models of fluid motion. Comput. Math. Appl. 60 (2010) 1440-1456.

[33] M. Marion and R. Temam, Navier-Stokes equations: Theory and approximation. Handb. Numer. Anal. VI (1998) 503-688. 
[34] C. Manica, M. Neda, M.A. Olshanskii and L. Rebholz, Enabling accuracy of Navier-Stokes-alpha through deconvolution and enhanced stability. ESAIM: M2AN 45 (2011) 277-308.

[35] P. Mininni, D. Montgomery and A. Pouquet, Numerical solutions of the three-dimensional magnetohydrodynamic $\alpha$ model. Phys. Rev. E 71 (2005) 1-11.

[36] R. Moser, J. Kim and N. Mansour, Direct numerical simulation of turbulent channel flow up to Re $=$ 590. Phys. Fluids 11 (1999) 943-945.

[37] A.P. Oskolkov, The uniqueness and solvability in the large of boundary value problems for the equations of motion of aqueous solutions of polymers. Zap. Naucn. Sem. Leningrad. Otdel. Mat. Inst. Steklov. (LOMI) 38 (1973) 98-136.

[38] A.P. Oskolkov, On the theory of unsteady flows of kelvin-voigt fluids. Zap. Nauchn. Sem. Leningrad. Otdel. Mat. Inst. Steklov. (LOMI) 115 (1982) 191-202. Boundary value problems of mathematical physics and related questions in the theory of functions, 14.

[39] L.G. Rebholz and M. Sussman, On the high accuracy NS- $\alpha$-deconvolution model of turbulence. Math. Models Methods Appl. Sci. 20 (2010) 611-633.

[40] L. Rebholz and S. Watro, A note on Taylor-eddy and Kavosnay solutions of NS- $\alpha$-deconvolution and Leray- $\alpha$-deconvolution models. J. Nonlinear Dyn. 2014 (2014) 1-5.

[41] M. Schäfer and S. Turek, The benchmark problem 'flow around a cylinder' flow simulation with high performance computers II. In vol. 52 of Notes on Numerical Fluid Mechanics. Edited by E.H. Hirschel. Braunschweig, Vieweg (1996) $547-566$.

[42] I. Stanculescu, Existence theory of abstract approximate deconvolution models of turbulence. Ann. Univ. Ferrara Sez. VII Sci. Mat. 54 (2008) 145-168.

[43] S. Stolz, N. Adams and L. Kleiser, The approximate deconvolution model for large-eddy simulations of compressible flows and its application to shock-turbulent-boundary-layer interaction. Phys. Fluids 13 (2001) 2985-3001.

[44] S. Stolz, N. Adams and L. Kleiser, An approximate deconvolution model for large-eddy simulations with application to incompressible wall-bounded flows. Phys. Fluids 13 (2001) 997-1015.

[45] S. Zhang, A new family of stable mixed finite elements for the 3d Stokes equations. Math. Comput. 74 (2005) 543-554. 